\title{
Solar magnetic fields as revealed by Stokes polarimetry
}

\section{Review Article}

\section{Author(s):}

Stenflo, Jan Olof

Publication date:

2013-11

Permanent link:

https://doi.org/10.3929/ethz-b-000072557

Rights / license:

In Copyright - Non-Commercial Use Permitted

Originally published in:

The Astronomy and Astrophysics Review 21(1), https://doi.org/10.1007/s00159-013-0066-3 


\title{
Solar magnetic fields as revealed by Stokes polarimetry
}

\author{
J.O. Stenflo
}

Received: 10 July 2013 / Published online: 21 September 2013

(C) Springer-Verlag Berlin Heidelberg 2013

\begin{abstract}
Observational astrophysics started when spectroscopy could be applied to astronomy. Similarly, observational work on stellar magnetic fields became possible with the application of spectro-polarimetry. In recent decades there have been dramatic advances in the observational tools for spectro-polarimetry. The four Stokes parameters that provide a complete representation of partially polarized light can now be simultaneously imaged with megapixel array detectors with high polarimetric precision $\left(10^{-5}\right.$ in the degree of polarization). This has led to new insights about the nature and properties of the magnetic field, and has helped pave the way for the use of the Hanle effect as a diagnostic tool beside the Zeeman effect. The magnetic structuring continues on scales orders of magnitudes smaller than the resolved ones, but various types of spectro-polarimetric signatures can be identified, which let us determine the field strengths and angular distributions of the field vectors in the spatially unresolved domain. Here we review the observational properties of the magnetic field, from the global patterns to the smallest scales at the magnetic diffusion limit, and relate them to the global and local dynamos.
\end{abstract}

Keywords Sun: atmosphere - Magnetic fields · Polarization · Dynamo · Magnetohydrodynamics (MHD)

\section{Historical background}

The discovery and classification by Joseph Fraunhofer of the absorption lines in the Sun's spectrum and the demonstration by Bunsen and Kirchhoff that such lines rep-

\section{J.O. Stenflo $(\varangle)$}

Institute of Astronomy, ETH Zurich, 8093 Zurich, Switzerland

e-mail: stenflo@ astro.phys.ethz.ch

J.O. Stenflo

Istituto Ricerche Solari Locarno, Via Patocchi, 6605 Locarno Monti, Switzerland 
resent "fingerprints" of chemical elements marked the birth of modern astrophysics. Spectral analysis became the standard tool of astronomers to determine the temperatures, densities, velocities, and chemical compositions of stellar atmospheres.

From the electromagnetic wave theory of Maxwell and Hertz it follows that the spectral radiation is not only characterized by its intensity, but also by its polarization. G.G. Stokes showed how the complete intensity and polarization information of a light beam could be described in a unified way in the form of a 4-vector, the Stokes vector. The first vector component represents the ordinary intensity, the second and third components describe linear polarization along two directions in the transverse plane, while the fourth component relates to the circular polarization.

This representation is very elegant and powerful, since it can describe any partially polarized light beam, and all the four vector components are given in the same units (intensity). Going from spectroscopy to spectro-polarimetry thus means that we increase the dimensionality of information space from 1-D to 4-D.

The presence of nonzero polarization implies a breaking of the symmetry in the source region. In reflection and scattering it is the relative directions of the incident and the reflected or scattered beams that break the symmetry. However, of greater diagnostic importance in astrophysics is the breaking of the spatial symmetry by a magnetic field. The Lorentz force induces a precession of the oscillating atomic dipole moment around the magnetic-field vector (Larmor precession), with the result that the transition frequencies between the different atomic levels get split into different components, which are polarized in ways that depend on the strength and orientation of the field. This effect, discovered by Pieter Zeeman in 1896, led to the discovery of magnetic fields in sunspots by Hale (1908).

In the usual theory of the Zeeman effect the split components superpose incoherently, i.e., they behave like independent lines. However, in scattering processes there are phase relations between the different magnetic $m$ substates of the excited state, a coherent superposition of quantum amplitudes that can be thought of as a "Schrödinger cat state", provided that the relative phases do not get scrambled by collisions during the life time of the excited state. In 1923 Wilhelm Hanle demonstrated experimentally how an imposed magnetic field that breaks the $m$ state degeneracy causes partial decoherence that increases to become complete with increasing strength of the field (Hanle 1924). His results provided guidance to the early development of quantum theory, since they represented a direct demonstration of the concept of linear superposition of quantum states, which is a cornerstone of quantum mechanics.

The effect of the magnetic field on the $m$ state interference and decoherence with the accompanying, observable polarization effects in the scattered radiation, is what is called the Hanle effect. The resulting polarization phenomena in the Sun's spectrum can be used to diagnose solar magnetic fields in parameter domains that are not accessible to the ordinary (incoherent) Zeeman effect. The Hanle and Zeeman effects are therefore highly complementary. A theoretical foundation for the application of the Hanle effect in astrophysics was established through the work of House (1970a, 1970b, 1971) Omont et al. (1973), Bommier and Sahal-Brechot (1978), and Stenflo (1978). The observational programs initially aimed at the diagnostics of magnetic fields in solar prominences (e.g. Leroy et al. 1977). 
Recent compilations of articles on various aspects of solar magnetism have been provided by Thompson et al. (2009) and Hasan and Rutten (2010). When the present review journal (Astron. Astrophys. Rev.) was founded, its first article was on the topic "Small-scale magnetic structures on the Sun" (Stenflo 1989). The diagnostic use of polarized radiation to explore solar magnetism has been the topic of a few monographs (Stenflo 1994b; del Toro Iniesta 2003; Landi Degl'Innocenti and Landolfi 2004) and has been the focus of a series of International Solar Polarization Workshops, which have taken place every three years since the first one in St. Petersburg, Russia, in 1995. The proceedings of these Workshops (six of them so far) provide a good account of the state of the art in the field (Stenflo and Nagendra 1996; Nagendra and Stenflo 1999; Trujillo Bueno and Sanchez Almeida 2003; Casini and Lites 2006; Berdyugina et al. 2009; Kuhn et al. 2011).

The present review tries to give a broad account of what we have learnt about solar magnetic fields from spectro-polarimetry by addressing a number of key topics, like the global and local dynamos, intermittency, and turbulence. By selectively focusing on these more general aspects, we have left out some other important topics, like the magnetic properties of sunspots, flares, or other active phenomena, which have received much attention in the solar physics literature. The selection of topics is biased by representing areas that have been of particular interest in the work of the present author. This choice has made it easier to present a coherent picture of the subject as opposed to trying to report on and give adequate credit to the diverse and often diverging world-wide contributions to the field.

The observed field properties that we are reviewing mainly reflect the conditions in the Sun's photosphere, since most diagnostics with Stokes polarimetry depends on the use of photospheric spectral lines. In future, attention will shift towards the chromosphere, transition region, and corona, but so far Stokes polarimetry of these regions have added relatively little to our understanding of the fundamental properties of the Sun's magnetic field.

Much of contemporary solar physics deals with the properties of solar magnetic fields, since they are responsible for all of solar and stellar activity and variability on intermediate time scales and govern space weather processes. Because the literature on all these various topics has grown immensely, the present review does not aim for any completeness in giving credit to all the important contributions in the field. In the selection of references we generally try to include references to the older discovery papers of the respective topics in addition to referring to more recent work, to avoid the impression that most of what we know comes from recent discoveries.

\section{Overview of Stokes polarimetry}

Electromagnetic radiation consists of an ensemble of wave packets (photons) with an electric vector that oscillates in the transverse plane (perpendicular to the propagation direction). Each such wave packet is $100 \%$ elliptically polarized, i.e., the trajectory of the tip of the electric vector describes an ellipse defined by three parameters: its size, the orientation angle of its major axis, and the ellipticity with sign (which determines the sense of rotation, left or right). These parameters are inconvenient to work 


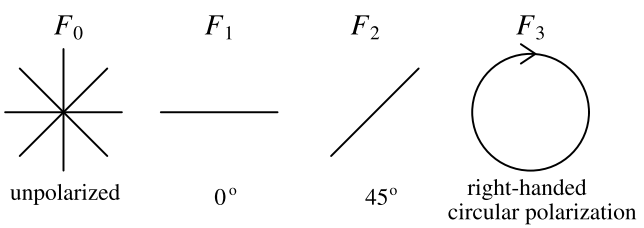

Fig. 1 Symbolic representation of four idealized filters used for the definition of the four Stokes parameters. Filter $F_{0}$ is empty, $F_{1}$ and $F_{2}$ transmit linear polarization oriented at $0^{\circ}$ and $45^{\circ}$, while $F_{4}$ transmits right-handed circularly polarized light. From Stenflo (1994b)

with, since they have different dimensions, and the polarization ellipse is incapable of describing partial polarization. The degree of polarization would have to be added as a fourth parameter to obtain a full description of polarized light.

Except for laser light the radiation that we encounter in nature represents an incoherent superposition of the $100 \%$ elliptically polarized wave packets of the ensemble. Since their phases are not correlated with each other, the superposition causes the degree of polarization of the ensemble to usually be much smaller than $100 \%$.

\subsection{Stokes parameters and Mueller calculus}

There are different formalisms for dealing with polarization, but the most convenient and powerful description that also relates directly to the measurement process is in terms of the four Stokes parameters, which can be combined into a 4-vector, the Stokes vector

$$
\mathbf{I}=\left(\begin{array}{c}
S_{0} \\
S_{1} \\
S_{2} \\
S_{3}
\end{array}\right) \equiv\left(\begin{array}{c}
I \\
Q \\
U \\
V
\end{array}\right) .
$$

There are different ways to define these parameters, but the operational definition in terms of the four ideal filters $F_{k}, k=0,1,2,3$ illustrated in Fig. 1 is directly related to the measurement process. If $I_{k}$ is the intensity that is measured by a detector placed behind filter $F_{k}$, then Stokes parameter $S_{k}$ is defined as

$$
S_{k}=2 I_{k}-I_{0}
$$

The inversion of this equation is

$$
I_{k}=\frac{1}{2}\left(I_{0}+S_{k}\right)
$$

If we would replace the three polarizing filters with their orthogonal versions (vertical polarization, $-45^{\circ}$ polarization, left-handed circular polarization), then the signs of the corresponding $S_{k}$ would change. From this it follows that Stokes $Q$ represents the difference between horizontal and vertical linear polarization, Stokes $U$ the difference between + and $-45^{\circ}$ linear polarization, Stokes $V$ the difference between right- and left-handed circular polarization. 
Note that all four Stokes parameters have the same dimension, intensity, although $Q, U$, and $V$ represent intensity differences. The intensity $I$ (proportional to the number of photons) can always be considered as the sum of two contributions:

$$
I=I_{u}+I_{p}
$$

where

$$
I_{p}=\sqrt{Q^{2}+U^{2}+V^{2}}
$$

represents $100 \%$ elliptically polarized light, while $I_{u}$ is completely unpolarized (with $Q=U=V=0$ ). The degree of polarization is then defined as

$$
p=\frac{I_{p}}{I_{u}+I_{p}}=\frac{\sqrt{Q^{2}+U^{2}+V^{2}}}{I} .
$$

$Q / I$ and $U / I$ represent the fractional linear polarizations in the horizontal and $+45^{\circ}$ directions, $V / I$ the fractional circular polarization.

The effect of a medium on the Stokes vector I can be described by the $4 \times 4$ Mueller matrix $\mathbf{M}$ :

$$
\mathbf{I}^{\prime}=\mathbf{M I}
$$

The medium may be of any kind, like a telescope system or a stellar atmosphere. If the medium is described as a sequence of consecutive components $i=1,2, \ldots, n$ (like a sequence of retarders, polarizers, or modulators in a telescope system, or a sequence of differential layers in a stellar atmosphere), each with its own Mueller matrix $\mathbf{M}_{i}$, then

$$
\mathbf{M}=\mathbf{M}_{n} \mathbf{M}_{n-1} \cdots \mathbf{M}_{2} \mathbf{M}_{1} .
$$

Index $i$ increases in the propagation direction (the order is essential).

Polarized radiative transfer can be formulated in a way similar to that of unpolarized radiative transfer. The main difference is the dimensionality of the problem. Instead of a scalar problem we have to deal with the transfer of a 4-vector, which is operated on by $4 \times 4$ matrices, which do not commute. This greatly enhances not only the complexity but also the richness of the problem and its solutions. In terms of diagnostics, we go from 1-D to 4-D information space. It is important to note that the information provided by the new dimensions $(Q, U$, and $V)$ cannot be derived from Stokes $I$, but each dimension provides a different diagnostic window to the universe. We will see many examples on this.

\subsection{Spectro-polarimeters}

As we saw in Sect. 2, images of the $Q, U$, and $V$ parameters are obtained by forming differences between images in orthogonal polarization states. There are two principal ways of forming such differences: (1) Recording the orthogonal polarization states simultaneously with a polarizing beam splitter. Advantage: Identical seeing effects in the two images, which subtract out when forming the difference. Disadvantages: 
The images fall on different detector areas with different gain tables, and it gets complicated when trying to record all Stokes parameters simultaneously $(Q, U$, and $V$ require six images). (2) Doing sequential switching of the polarization state (modulation of the beam). Advantages: The same detector area and gain table is used for all the polarization states, and one can modulate all the Stokes parameters with a single beam. Disadvantage: The images are not simultaneous and may therefore be subject to different seeing distortions, which generate spurious polarization signatures in the difference images.

If one can modulate faster than the seeing frequencies, then the modulation approach is superior to the beam splitter approach. A breakthrough in the mapping of solar magnetic fields came with the introduction by Babcock (1953) of the solar magnetograph, which used electro-optical modulation of the circular polarization. The detector was a photomultiplier in the spectrograph focal plane, receiving the light selected by exit slits in the wings of a spectral line sensitive to the Zeeman effect, and the demodulation was performed with a lock-in amplifier. This technique was extended by Stepanov and Severny (1962) to record all Stokes parameters and thereby produce maps of the vector magnetic field. Since the detectors used at that time were basically 1-pixel devices (photomultipliers), images had to be built up by mechanical scanning. Line profiles were not recorded, only selected wavelength bands usually positioned in the wing of a spectral line.

Stokes polarimeters that could simultaneously record the full line profiles of the four Stokes parameters were developed in the 1970s with various types of detectors (photographic plates or linear arrays), beam splitters, and modulators (Harvey et al. 1972; Wittmann 1973; House et al. 1975; Baur et al. 1980). This development transformed spectroscopy from 1-D (intensity) to 4-D (the four Stokes parameters).

The next technological advance came in the 1980s with the introduction of 2-D CCD type detectors in astronomy. While they allow for typically a million simultaneous image elements (pixels) in contrast to the 1-pixel photomultiplier detectors, the readout is slow and therefore seemed to be incompatible with fast modulation. This is of no particular concern for magnetographs and polarimeters in space, like SOHO/MDI (Scherrer et al. 1995), or the various polarimetric instruments on the Hinode spacecraft like SOT/SP (Kosugi et al. 2007; Suematsu et al. 2008; Tsuneta et al. 2008), because of the absence of seeing outside the terrestrial atmosphere. In the case of ground-based observations, however, seeing noise becomes a limiting factor when the modulation is not fast enough.

The apparent incompatibility problem between the slow readout of large-area CCD detectors and fast polarization modulation found a solution with the ZIMPOL (Zurich Imaging Polarimeter) technology (Povel 1995, 2001; Gandorfer et al. 2004). Instead of trying to do readout at the modulation rate, the photo charges are shifted between the exposed areas and fast hidden buffers in synchrony with the modulation. Since CCD detectors do not have storage areas below the pixel layer, the buffer storage is created by depositing in the manufacturing process a mask on the sensor such that for each group of four pixel rows, one is open, while the other three are hidden behind the mask for use as buffers. The photo charges can be laterally shifted at fast rates and cycled through the four rows in synchrony with the modulation at $\mathrm{kHz}$ rates. This implies that we create four simultaneous image planes within the same CCD sensor, each of which represents a different state of polarization. 
To avoid light losses on the masked detector area an array of cylindrical microlenses is mounted on top of the sensor. Each microlens has a width of four pixel rows and ensures that all light falling on it is directed to the unmasked pixel row. After temporal integration over a large number (e.g. $10^{4}$ ) modulation cycles, all four image planes are read out. Through linear combinations between the image planes and use of the polarization calibration information, simultaneous images of the four Stokes parameters are obtained. The fractional polarization images $Q / I, U / I$, and $V / I$ are free from gain-table effects (flat-field errors), since the identical pixels have been used to expose the four image planes. Therefore the individual pixel gain factors divide out completely when forming the fractional polarization. This is a main reason why we for ZIMPOL observations do not represent the observed polarization images in terms of $Q, U$, and $V$, but instead in terms of these quantities divided by Stokes $I$. Flat-fielding is only needed for the Stokes $I$ image.

With this technology the two main noise sources get eliminated, seeing noise (because we modulate much faster than the seeing frequencies) and gain-table noise (which divides out in the fractional polarizations). In practice the polarimetric sensitivity is only limited by the Poisson statistics of the number of collected photo charges, which depends on the photon collecting area of the telescope, the optical transmission and detector efficiencies, the effective integration time, and the spatial and spectral resolutions. Optimizing these parameters ZIMPOL routinely achieves a polarimetric precision of $10^{-5}$ in combination with high spectral resolution.

\subsection{Trade-offs}

With a perfect polarimeter the precision is limited by the number of collected photons. It is a common misunderstanding that we always receive enough photons from the Sun because it is so close to us. The number of photons from a resolved stellar disk per diffraction-limited angular resolution element is independent of stellar distance and only depends on the effective temperature of the stellar surface. If we want to do polarimetry with an angular resolution at the telescope diffraction limit, then the solar observations are severely photon starved. It is never possible to independently optimize the four basic observing parameters spectral, spatial, and temporal resolution and polarimetric accuracy, major trade-offs are always necessary. It is common that solar astronomers give priority to the highest possible spatial resolution to probe the smallest solar structures, but this can only be done at the expense of polarimetric precision and/or spectral resolution. Temporal resolution cannot be chosen independently of angular resolution, since smaller structures evolve faster, and the effective integration time must be shorter than the evolutionary time scale.

Figure 2 gives an overview of the trade-offs in the mentioned 4-D observational parameter space. We see that with a 4-m solar telescope like the future ATST it is theoretically possible to reach a polarimetric precision of $10^{-4}$ in combination with a spatial resolution of $100 \mathrm{~km}$, but for a precision of $10^{-5}$, which is possible with a polarimeter like ZIMPOL and needed for explorations of the Second Solar Spectrum and the Hanle effect, the angular resolution cannot be better than about 1 arcsec even with such a large telescope. 


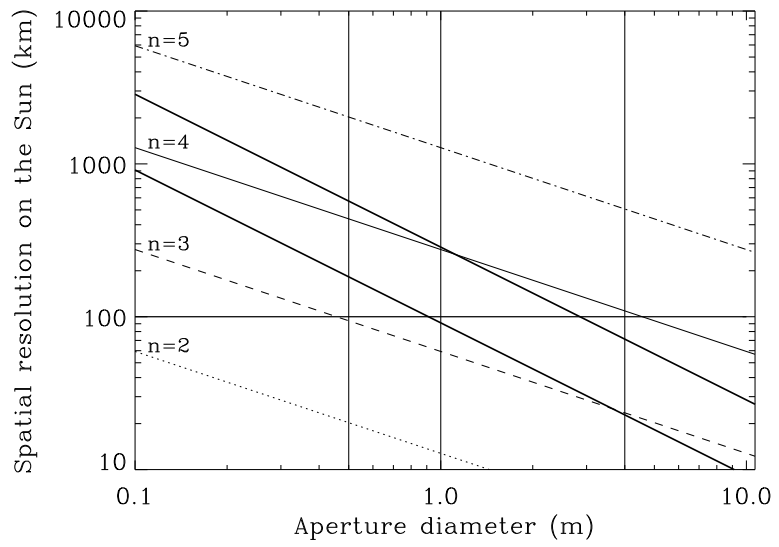

Fig. 2 Trade-offs between spatial resolution and polarimetric precision as a function of telescope aperture size, assuming a spectral resolution of 300000 and a maximum integration time that is limited by the evolutionary time scale for the given spatial scale. The thin slanted lines labeled by parameter $n$ show where the polarimetric precision is $10^{-n}$. The thick slanted lines give the telescope diffraction limit for wavelengths $1.56 \mu \mathrm{m}$ (upper line) and $5000 \AA$ (lower line). The vertical lines mark aperture sizes $0.5,1$, and $4 \mathrm{~m}$. From Stenflo (1999)

\section{Zeeman effect}

Since its first application in astrophysics little more than a century ago (Hale 1908), the Zeeman effect has been the prime tool to gain information about cosmic magnetic fields. With modern imaging polarimeters we can produce simultaneous images of the four Stokes parameters, either with narrow-band filters to obtain 2-D maps of the solar surface in a selected wavelength band, or with spectrographs to obtain detailed Stokes line profiles for each resolution element along the spectrograph slit. An example of such simultaneous spectral images of the four Stokes parameters obtained with high spatial resolution $(0.3 \mathrm{arcsec})$ with the SOT/SP instrument on the Hinode spacecraft is shown in Fig. 3. The spectral structures in Stokes $V$ are due to the longitudinal Zeeman effect and generally have anti-symmetric profile shapes, while the structures in $Q$ and $U$ are caused by the transverse Zeeman effect and have symmetric profile shapes. The polarized spectra reveal the existence of highly structured magnetic fields everywhere on the quiet Sun. The intensity variations and line wiggles seen in Stokes $I$ are due to the solar granulation.

An intuitive understanding of such Zeeman-effect spectra can be developed in terms of the basic symmetry properties of the Stokes profiles and their different sensitivities to the magnetic field. The essential properties can be sufficiently exposed by considering the two idealized cases of a homogeneous magnetic field oriented (a) along the line of sight, and (b) perpendicular to the line of sight. No assumptions or explicit representations for the model atmosphere are needed (cf. Stenflo 1994b).

Through a suitable choice of basis polarization vectors, the four coupled transfer equations for the four Stokes parameters decouple (the problem gets diagonalized). For case (a) we choose right- and left-handed polarized unit vectors, corresponding to the polarization of the two $\sigma$ components of the longitudinal Zeeman pattern. For case (b) we choose two unit vectors linearly polarized along and perpendicular to the 
Fig. 3 Example of Stokes spectra of the quiet-sun disk center for the spectral window with the two Fe I 6301.5 and $6302.5 \AA$ A lines. The recording was made with the Hinode SOT/SP instrument in deep mode (9.6 s integration time) on February 27, 2007. The spatial resolution is about $230 \mathrm{~km}$ on the Sun. From Lites et al. (2008)

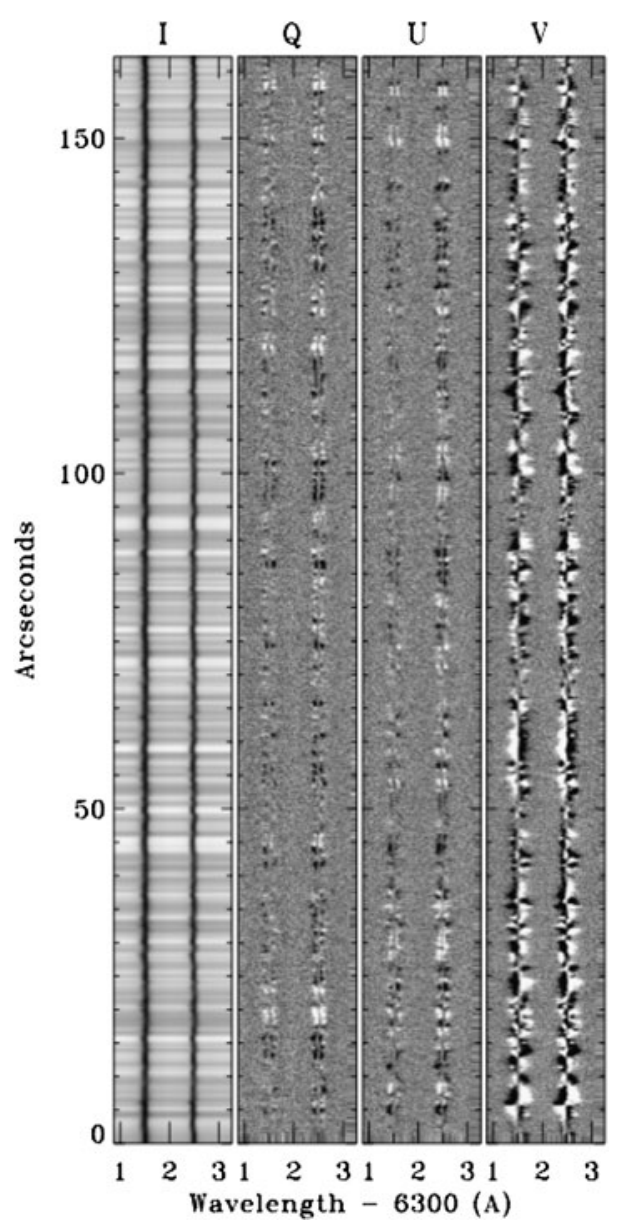

direction of the transverse magnetic field, corresponding to the polarization of the $\pi$ and $\sigma$ components of the transverse Zeeman pattern. The decoupling of the transfer equations implies that the atmosphere decouples into two mutually independent, noninteracting atmospheres.

Let us first consider case (a). If $I_{0}(\Delta \lambda)$ represents the emergent spectrum from each atmosphere in the limit of vanishing magnetic field, then in the presence of a homogeneous longitudinal field with Zeeman splitting $\Delta \lambda_{H}$ the emergent spectra $I_{ \pm}$ from the two atmospheres can simply be expressed as

$$
I_{ \pm}(\Delta \lambda)=I_{0}\left(\Delta \lambda \mp \Delta \lambda_{H}\right)
$$

This is an exact result for a normal Zeeman triplet, valid for all field strengths, line strengths, and static model atmospheres. 
Transforming back to the standard Stokes system (which uses Cartesian basis vectors) we get

$$
\begin{aligned}
I & =\frac{1}{2}\left(I_{+}+I_{-}\right) \approx I_{0}+\frac{1}{2}\left(\Delta \lambda_{H}\right)^{2} \partial^{2} I_{0} / \partial \lambda^{2}+\cdots \\
V & =\frac{1}{2}\left(I_{+}-I_{-}\right) \approx-\Delta \lambda_{H}\left[\partial I_{0} / \partial \lambda+\frac{1}{6}\left(\Delta \lambda_{H}\right)^{2} \partial^{3} I_{0} / \partial \lambda^{3}+\cdots\right] .
\end{aligned}
$$

For weak magnetic fields (when the Zeeman splitting $\Delta \lambda \ll$ the spectral line width)

$$
\begin{aligned}
I & \approx I_{0}, \\
V & \approx-\Delta \lambda_{H} \partial I_{0} / \partial \lambda,
\end{aligned}
$$

where

$$
\Delta \lambda_{H}=z g \lambda^{2} B
$$

and $g$ is the Landé factor. $z=4.67 \times 10^{-13} \AA^{-1} \mathrm{G}^{-1}$. This implies that

$$
V \sim B \frac{\partial I}{\partial \lambda} .
$$

Stokes $V$ therefore has a profile shape that mimics the anti-symmetric gradient of the Stokes $I$ spectrum, and the $V$ amplitude is proportional to the field strength. Beyond the weak-field regime, when the Zeeman splitting is no longer small in comparison with the line width, the relation between Stokes $V$, field-strength $B$, and intensity profile $I$ becomes nonlinear (Zeeman saturation). This nonlinearity can be used to infer the existence and strengths of intermittent flux concentrations at scales far smaller than the telescope resolution, as we will see in Sect. 8.2.

For photospheric lines there are generally fluctuating and systematic deviations from the proportionality described by Eq. (13), for two reasons: (1) The photosphere is dynamic with correlated magnetic- and velocity-field gradients along the line of sight, which leads to Stokes $V$ asymmetries that do not obey Eq. (13) (cf. Illing et al. 1975; Auer and Heasley 1978; Stenflo et al. 1984), and (2) much of the photospheric flux is in collapsed, strong-field form, while photospheric lines in general have small line widths, with the consequence that lines with large Landé factors are affected by Zeeman saturation (deviation from linearity of the field-strength dependence).

For the broad chromospheric lines, however, the $V$ profile shape is nearly identical to the shape of $-\partial I / \partial \lambda$, as illustrated in Fig. 4 for the well-known Na I $D_{1} 5896 \AA$ line. The numerous telluric water vapor lines that surround the sodium line are conspicuous in the $-\partial I / \partial \lambda$ spectrum, but as they are unpolarized they are entirely absent in the Stokes $V$ spectrum.

Let us now turn to case (b), a transverse magnetic field in a static atmosphere. As before, the atmosphere can be treated as composed of two mutually noninteracting atmospheres, one relating to the transfer of the $\pi$ component, the other related to the two $\sigma$ components. However, in contrast to the longitudinal case the two $\sigma$ components now have the same polarization state. They therefore interact in the radiative-transfer process if the medium is optically thick. To avoid this complication 


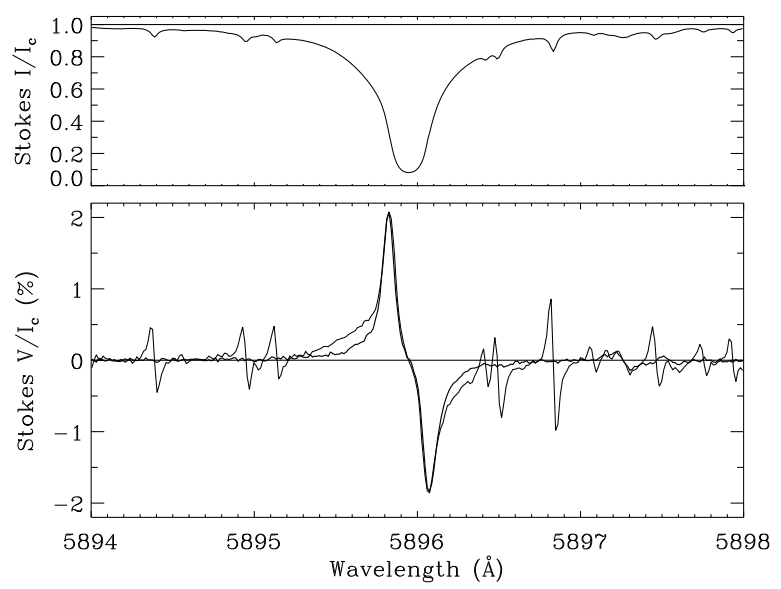

Fig. 4 Example of a small spectral section around the chromospheric Na I $D_{1} 5896 \AA$ line from the Stokes $V$ atlas of a strong plage at disk center recorded on April 30, 1979, with the FTS (Fourier transform spectrometer) of the NSO McMath-Pierce facility at Kitt Peak. In the bottom panel the Stokes $V$ spectrum is shown (thick solid line) with the intensity gradient $-\partial I / \partial \lambda$ superposed (thin solid line) after it has been normalized to the $V$ amplitude. Note the numerous spectral features in $-\partial I / \partial \lambda$ from water vapor in the terrestrial atmosphere. These telluric features are completely absent in the $V$ spectrum. Adapted from Stenflo et al. (1984)

for the present conceptual discussion, let us assume that we are dealing with weak (optically thin) spectral lines. If again $I_{0}(\Delta \lambda)$ represents the emergent spectrum from each atmosphere in the limit of vanishing magnetic field, then in the presence of a homogeneous transverse field the emergent spectra $I_{\pi}$ and $I_{\sigma}$ from the two atmospheres become

$$
\begin{aligned}
& I_{\pi}=I_{0}(\Delta \lambda), \\
& I_{\sigma}=\frac{1}{2}\left[I_{0}\left(\Delta \lambda-\Delta \lambda_{H}\right)+I_{0}\left(\Delta \lambda+\Delta \lambda_{H}\right)\right] .
\end{aligned}
$$

In the standard Stokes system with the positive Stokes $Q$ direction defined along the magnetic field, we get

$$
\begin{aligned}
& I=\frac{1}{2}\left(I_{\pi}+I_{\sigma}\right) \approx I_{0}+\frac{1}{4}\left(\Delta \lambda_{H}\right)^{2} \partial^{2} I_{0} / \partial \lambda^{2}+\cdots, \\
& Q=\frac{1}{2}\left(I_{\pi}-I_{\sigma}\right) \approx-\frac{1}{4}\left(\Delta \lambda_{H}\right)^{2} \partial^{2} I_{0} / \partial \lambda^{2}+\cdots .
\end{aligned}
$$

For weak fields

$$
Q \sim B^{2} \frac{\partial^{2} I}{\partial \lambda^{2}} .
$$

These expressions show that the linear polarization profiles are symmetric and in the weak-field and weak-line limit mimic the second derivative of the Stokes $I$ spectrum. Note in particular that for weak fields the linear polarization scales with $B^{2}$, in contrast to the circular polarization, which is linear in $B$. 
For a field that is oriented with azimuth angle $\chi$ with respect to the defined $Q$ direction,

$$
\begin{aligned}
& Q \sim B^{2} \cos 2 \chi \frac{\partial^{2} I}{\partial \lambda^{2}}, \\
& U \sim B^{2} \sin 2 \chi \frac{\partial^{2} I}{\partial \lambda^{2}} .
\end{aligned}
$$

If we now go to the general case when the magnetic-field vector has an arbitrary inclination $\gamma$ with respect to the line of sight, then all the previous equations remain valid if we replace $B$ in the expressions for Stokes $V$ with $B \cos \gamma$ (the line-of-sight component) and (in the optically thin limit) replace $B^{2}$ in the expressions for $Q$ and $U$ by $B^{2} \sin ^{2} \gamma$ (the square of the transverse field component). Although this $\gamma$ dependence is not exact in the case of strong lines (due to transfer effects in optically thick media and magneto-optical effects), it remains a good approximation. However, the $Q$ and $U$ profile shapes will differ substantially from the $\partial^{2} I / \partial \lambda^{2}$ shape when the lines are no longer optically thin.

While the circular polarization is related linearly to the line-of-sight component $B_{\|}$of the field according to Eq. (13), the linear polarization scales with the square of the transverse component, in other words with the magnetic energy density of the transverse component, according to Eq. (17). Thus with the weak-field approximation, which is valid for practically all lines when $B \lesssim 0.5 \mathrm{kG}$,

$$
\begin{aligned}
& B_{\|} \sim V, \\
& B_{\perp} \sim\left[Q^{2}+U^{2}\right]^{1 / 4} .
\end{aligned}
$$

In addition to its nonlinear field dependence, the linear polarization has much less field sensitivity than the circular polarization, which makes the transverse field highly susceptible to noise. As an example we consider the state-of-the-art Hinode SOT/SP recording that was illustrated in Fig. 3. With radiative-transfer modeling of the Fe I $6301.5 \AA$ line the proportionality constants in Eq. (18) can be estimated to be 29.4 for the longitudinal and 184 for the transverse equation, provided that the Stokes parameters are expressed in $\%$ of the continuum intensity and the field strength in $\mathrm{G}$ (Stenflo 2011). The measured 1- $\sigma$ noise in Stokes $V$ is $0.047 \%$, while it is somewhat smaller, $0.035 \%$, in each of $Q$ and $U$. When however the Gaussian noise distributions are translated into noise distributions for $B_{\|}$and $B_{\perp}$, we get the distributions shown in Fig. 5. In spite of the smaller noise in the linear polarization, the noise in the transverse field is larger by approximately a factor of 25 . What is much worse, however, is the profoundly non-Gaussian nature of the noise distribution due to the nonlinear field dependence of the linear polarization. This makes it very difficult to obtain any reliable determinations of vector magnetic fields in quiet solar regions, where the magnetic fluxes are weak. If for instance in disk center observations only a tiny fraction of the noise in $B_{\perp}$ would be mistaken for a solar signal, then the field would appear to be more horizontal than it really is.

Another serious consequence of the nonlinear response of the linear polarization to the magnetic field is a model dependence of the interpretation of spatially averaged 


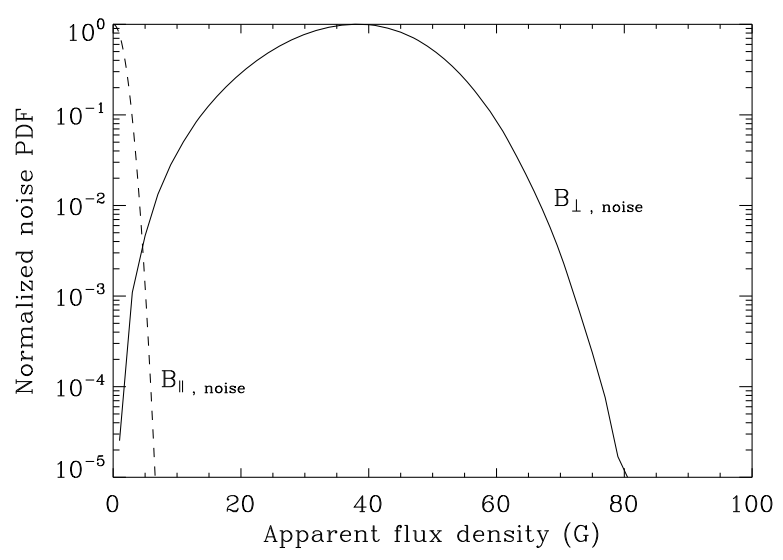

Fig. 5 Histograms of the noise in the deep-mode Hinode SOT/SP observations, converted from polarization to field-strength units using the weak-field approximation. The measured polarization noise is Gaussian with standard deviations $0.035 \%$ for Stokes $Q$ and $U, 0.047 \%$ for $V$. Although the noise in the linear polarization is smaller, it translates to much larger apparent field strengths $B_{\perp \text {, noise }}$ than the apparent field strengths $B_{\| \text {,noise }}$ of the circular polarization. Adapted from Stenflo (2011)

quantities. We know that the magnetic structuring continues far beyond the resolution limit of current telescopes, where we expect to have both collapsed kG-type field concentrations and turbulent fields that are tangled on small scales. In nearly all cases (except for large structures like sunspots) the pixels of the detector are much larger than the solar magnetic structures. Averaging over a pixel is not a serious problem when the polarimetric response to the magnetic field is linear, as it approximately is for the circular polarization. Regardless of the nature of the subpixel structuring, the pixel average of Stokes $V$ is proportional to the line-of-sight component of the total magnetic flux through the pixel area, or, equivalently, to the average strength of the line-of-sight component.

A similar type of interpretation is not possible for the transverse magnetic field. The pixel average of $Q$ or $U$ is in a first approximation proportional to the average magnetic energy density of the transverse component, but a translation of average energy density to average field strength is not possible without an assumed model for the subpixel structuring, including the subpixel distributions of field strengths, azimuths, and inclinations.

The determination of vector magnetic fields is therefore not a technical problem like how to develop efficient algorithms for Stokes inversion, but it is more fundamental, namely how to combine one component (along the line of sight) that represents flux with another component (the transverse one) for which the flux is not an observable. The problem is aggravated by the circumstance that the noise in the line-of-sight flux is Gaussian and very small in comparison with the non-Gaussian transverse field noise.

Although attempts to map vector magnetic fields on the Sun have been made for more than half a century (starting with Stepanov and Severny 1962), it is not surprising that most magnetic-field work has focused on mapping and exploring the lineof-sight component through recordings of the circular polarization. With the advent 
of sensitive imaging polarimeters that can record the full Stokes line profiles with good spatial resolution, as illustrated in Fig. 3, there has been a revived interest in the determination of vector magnetic fields, but there has been a tendency to downplay the fundamental limitations just mentioned when doing formal Stokes inversions to generate maps of the vector magnetic field.

\section{Hanle effect}

Polarization is related to some symmetry-breaking process. In the case of the Zeeman effect it is the magnetic field that breaks the spatial symmetry. In the absence of magnetic fields the symmetry can be broken in a scattering process, depending on the directional relations between the incident and scattered radiation. The oscillating electric vector of the incident light excites an oscillating dipole moment in the scattering atomic system. The emitted dipole radiation depends on how the dipole was excited.

\subsection{Scattering polarization}

The blue sky is linearly polarized by molecular Rayleigh scattering. Similarly the solar spectrum is linearly polarized by scattering processes in the Sun's atmosphere. In classical dipole scattering the polarization reaches $100 \%$ when the scattering angle is $90^{\circ}$, but on the Sun the polarization is much smaller because we average over a wide range of scattering angles. The angular distribution of the incident radiation must be anisotropic in order to generate any net polarization. For a spherically symmetric Sun (disregarding local inhomogeneities) the anisotropy expresses itself as limb darkening if the distribution has a net preference for the vertical direction. For symmetry reasons the scattering polarization vanishes at disk center and increases monotonically as we approach the limb. The emitted radiation is polarized with the electric vector oriented perpendicular to the radius vector (parallel to the nearest solar limb).

The idea of looking for scattering polarization on the Sun is old (e.g. Öhman 1929). The first survey of the scattering polarization throughout the solar spectrum (Stenflo et al. 1983a, 1983b), from the deep UV (near the atmospheric cut-off around $3165 \AA$ ) to the near infrared (up to about $9950 \AA$ ) revealed a wealth of unfamiliar polarized spectral structures, for instance the feature that extends over more than $200 \AA$ around the Ca II K and H lines at 3933 and $3968 \AA$ and which is caused by quantum interference between the $J=3 / 2$ and $1 / 2$ upper states of the $\mathrm{K}$ and $\mathrm{H}$ transitions (Stenflo 1980). It was like uncovering an entirely new and unfamiliar spectrum and one had to start over again to identify the various structures and their underlying physical mechanisms. This prompted V.V. Ivanov of St. Petersburg to introduce the now established name Second Solar Spectrum for the linearly polarized spectrum that is exclusively caused by coherent scattering processes (Ivanov 1991).

The polarimetric noise in this initial survey was of order $10^{-3}$. Only the most prominent polarizing lines protruded well above this level, but it was clear that they represented only the "tips of the icebergs". With the implementation of the ZIMPOL technology in the 1990s (see Sect. 2.2), with which the noise level could be reduced 
to $10^{-5}$, the full wealth of structures in the Second Solar Spectrum became accessible to observation (Stenflo and Keller 1996, 1997).

Scattering is coherent and a source of linear polarization if there is a phase relation between the excitation and emission process, in other words, if the emitting particle retains a "memory" about how it was excited. This memory can be erased by collisions during the excited state, which scramble the phase and therefore depolarize. There is a competition between the radiative decay rate and the collisional rate. The fraction of emission processes that are undisturbed by collisions represents coherent, polarizing scattering.

\subsection{Principles of the Hanle effect}

The coherent scattering process can also be affected by an external magnetic field, which causes modifications of the scattering polarization. It is the set of magnetically induced alterations of the scattering polarization that we refer to as the Hanle effect, discovered by Wilhelm Hanle in Göttingen in 1923 (Hanle 1924). An intuitive understanding of the Hanle effect is provided by a classical description in terms of the electric dipole oscillations of the scattering atom, as illustrated in the left portion of Fig. 6. The unpolarized incident radiation induces dipole oscillations in the transverse plane. Seen by an observer receiving light scattered at $90^{\circ}$, these oscillations are linear and perpendicular to the scattering plane. In the absence of a magnetic field we would then get $100 \%$ linear polarization along this direction (which is assumed to be the vertical direction in Fig. 6). If we introduce an external magnetic field along the scattering direction, the damped oscillator will precess around the field and describe a rosette pattern. We have a competition between the radiative damping rate and the rate of Larmor precession, which is proportional to the magnetic-field strength. For the three rosette patterns illustrated in Fig. 6 the field strength increases from left to right. If the precession rate is much smaller than the damping rate, the emission process gets completed before much precession has had time to take place. Then the scattering polarization will not deviate much from the nonmagnetic case. If on the other hand the precession rate is much larger than the damping rate, the orientations of the dipole oscillations get randomized, resulting in unpolarized radiation. In the intermediate case, when the two rates are comparable, the net effect on the scattered radiation is rotation of the plane of linear polarization combined with partial depolarization. The effect on the Stokes $Q$ and $U$ parameters is found through Fourier transformation of the rosette patterns.

The right side of Fig. 6 shows an example of what the observational signatures of the Hanle effect look like in the Sun's spectrum. The recording was made with the ZIMPOL polarimeter in a weakly magnetic region near the solar limb with the spectrograph slit oriented perpendicular to the radius vector (parallel to the nearest limb), and with positive Stokes $Q$ defined as linear polarization with the electric vector oriented along the slit. The spectral window is centered around the Ca I $4227 \AA$ line, which happens to be the line that exhibits the largest scattering polarization in the whole visible solar spectrum. The core of this line is formed in the low chromosphere well above the temperature minimum. In the absence of magnetic fields, scattering polarization would only show up as positive $Q$ with no variations along 
Hanle effect

Ca | $4227 \AA$, a chromospheric line

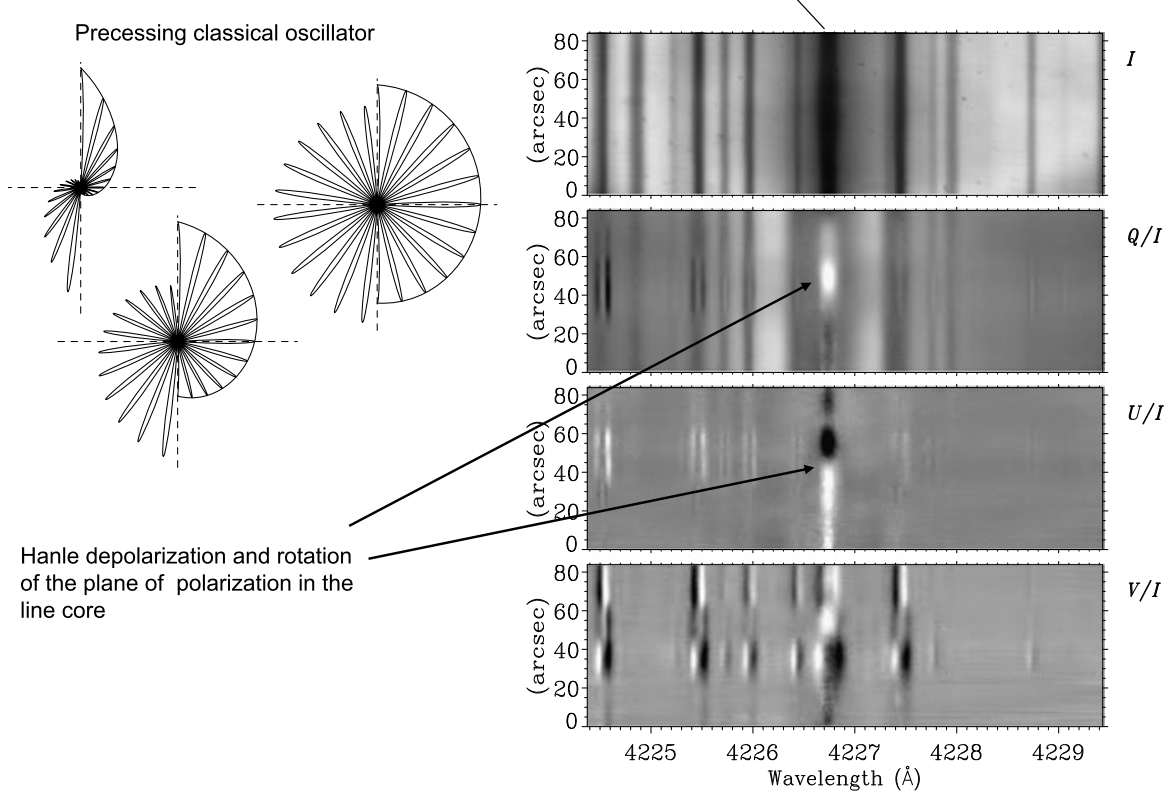

Fig. 6 Illustration of the Hanle effect, from Stenflo (2010b). The three rosette patterns to the left show the classical vector trajectories of a damped dipole oscillator that is precessing around a magnetic field that is oriented along the line of sight, for three values of the field strength, increasing from left to right. When converted to the Fourier domain the net effect of the magnetic field on the scattering polarization in the Doppler core of the considered spectral line is to depolarize and rotate the plane of polarization, as indicated by the spectral signatures seen in the $Q / I$ and $U / I$ panels to the right for the Ca I $4227 \AA$ line. The recording was made on March 9, 2002, at disk position $\mu=0.2$ near the heliographic S pole with ZIMPOL-2 at the NSO McMath-Pierce facility at Kitt Peak

the slit, while $U$ and $V$ would be zero. All the signatures that we see in the fractional circular polarization $(V / I)$ are due to the longitudinal Zeeman effect, while the symmetric doublet patterns seen in the surrounding blend lines in the fractional linear polarization $(Q / I$ and $U / I)$ are due to the transverse Zeeman effect. In the core of the strong $4227 \AA$ line, however, we notice a qualitatively completely different type of signature that has nothing to do with the transverse Zeeman effect but is due to the Hanle effect and varies along the slit because of spatially structured magnetic fields. The variations of $Q / I$ along the slit are caused by spatially varying Hanle depolarization, while the signatures in $U / I$ are due to spatially varying Hanle rotation of the polarization plane.

Note that there are also two bright bands in $Q / I$ on either side of the 4227 line core, which are due to nonmagnetic scattering polarization in the wings of this chromospheric line. These bands show no variations along the slit and are absent in $U / I$. The reason for this behavior is that the effectiveness of the Hanle effect is limited to the Doppler cores of spectral lines but is absent in the wings, where the scattering polarization behaves like in the nonmagnetic case. 
In quantum mechanics the Hanle effect can be understood in terms of the coherent superposition of the partially split magnetic $m$ substates. In a scattering process that is undisturbed by collisions, the excited, intermediate state represents a coherent superposition (with mutual phase relations) of the $m$ states (a "Schrödinger cat state"). It is the quantum interferences between the $m$ states that give rise to the polarization phenomena that represent the Hanle effect. A good overview of the physics and diverse applications of the Hanle effect is provided in Moruzzi and Strumia (1991).

The polarized scattering experiments in 1923-1924 that led to the discovery of the Hanle effect (Hanle 1924) played a key role in the early conceptual development of quantum mechanics, since they demonstrated experimentally the principle of linear superposition of atomic states with quantum interference and the partial decoherence caused by external magnetic fields that break the symmetry. The theoretical edifice of quantum mechanics was built on these concepts.

The Second Solar Spectrum is the playground for the Hanle effect, since it represents the spectrum that is exclusively due to coherent scattering, and it is the magnetic-field modifications of this spectrum that constitutes the Hanle effect. The exploitation of the rich potential of the Hanle effect requires a good understanding of the Second Solar Spectrum and the underlying physical mechanisms. A highprecision Atlas of the Second Solar Spectrum from the deep UV to the near infrared as recorded with the ZIMPOL polarimeter has been produced by Gandorfer (2000, 2002 , 2005). For an overview of the various types of physical effects revealed by this spectrum, like $J$ state interference, scattering at molecules and rare earth elements, continuum polarization, hyperfine structure and isotope effects, optical pumping, and still unexplained enigmatic physics, see Stenflo (2004).

The Hanle and Zeeman effects are highly complementary and diagnose different aspects of solar magnetism, both because of their different field sensitivities and their different symmetry properties. In the case of the Zeeman effect the field-strength sensitivity depends on the ratio between the Zeeman splitting and the line width, which is mainly determined by the Doppler width. In the case of the Hanle effect the sensitivity depends on the ratio between the Zeeman splitting and the damping width. Since the radiative damping width is smaller than the Doppler width by typically a factor of 25, the Hanle effect is sensitive to correspondingly weaker fields. This is of particular importance for the diagnostics of chromospheric magnetic fields, which are weak while the chromospheric spectral lines are generally broad.

\subsection{Hanle effect signatures of turbulent fields}

The Zeeman effect is blind to a turbulent field that has mixed polarities on a scale much smaller than the spatial resolution of the telescope, because the polarization contributions of the opposite polarities cancel each other. The Hanle rotation of the plane of polarization has a similar type of cancelation, but the Hanle depolarization effect does not, because it responds to the field with only one "sign" (depolarization).

This difference between the Hanle and Zeeman effects has its origin in the different symmetry properties of the Hanle effect in its dependence on the field orientation. Maximum Hanle depolarization is obtained when the magnetic field is oriented along the line of sight. The depolarization becomes complete in the strong-field limit, regardless of the field polarity. If the field is vertical and we assume that the illumination 
of the scattering atomic system is axially symmetric around the field vector, then the Hanle effect vanishes and the scattering polarization behaves like in the nonmagnetic case, regardless of the magnitude of the field strength. For a quantitative interpretation of the measured Hanle depolarization in terms of field strengths we therefore need some information or assumption about the field orientation.

This need can be satisfied if we interpret the observations not in terms of individual magnetic elements, but in terms of ensembles of unresolved elements within each spatial resolution element. The assumption that such ensembles of unresolved structures really exist on the Sun is validated by the behavior of the scattering polarization as observed in photospheric spectral lines: there is an absence of significant Hanle rotation effects (implying the existence of subresolution cancelation effects), at the same time as one finds very substantial Hanle depolarization that does not seem to fluctuate much on resolved spatial scales. This points to the real existence of a statistically nearly invariant ocean of unresolved structures that are much smaller than the resolved scales, so that the ensemble averages over each resolution element do not vary significantly.

Let us note that this behavior only applies to photospheric but not to chromospheric spectral lines. For the chromospheric lines the Hanle rotation and depolarization effects are of similar magnitude, as we expect when the fields are resolved. Furthermore, these effects are found to exhibit large fluctuations on the resolved spatial scales. Therefore our discussion of the turbulent Hanle effect does not apply to the chromosphere, it is exclusively a property of the photosphere.

Observations of the Hanle depolarization effect reveal that regions that look entirely empty as voids in high-resolution magnetograms cannot be voids at all but must be seething with an ocean of turbulent fields that carry a highly significant amount of magnetic flux and energy. As the smallest scales of the resolved domain are only slightly larger than the transition scale of about $100 \mathrm{~km}$ between the optically thick and thin regimes, we can conclude that the great majority of the flux elements that contribute to the observed Hanle depolarization are optically thin. This is the microturbulent regime, in which we can do direct ensemble averaging over the Hanle scattering phase matrix before solving the radiative-transfer problem (Stenflo 1982).

For the conversion of Hanle depolarization to field strength we need to characterize our field ensemble with a single free parameter that is uniquely determined by the single observational constraint (the observed depolarization). The simplest model is to assume that the ensemble is characterized by a single turbulent field strength $B_{t}$, that the angular distribution of field vectors is axially symmetric around the vertical direction, and then examine how the assumption for the distribution of inclination angles affects the result.

Two special types of distributions are of particular interest: an isotropic distribution, and a flat distribution that is confined to the horizontal plane. Figure 7 illustrates how the resulting Hanle depolarization depends on field strength $B_{t}$ for these two cases. The field strength sensitivity is greatest where the Larmor precession rate is comparable in magnitude to the inverse life time of the excited state. The difference between the two curves in Fig. 7 is not particularly large, which indicates that the interpretation is not very sensitive to the assumptions for the angular distribution. In general there is no good physical justification for the choice of the flat distribution 
Fig. 7 Hanle depolarization factor due to a turbulent magnetic field of strength $B_{t}$. The parameter $B_{0}$ depends on the atomic transition and represents the field strength for which the Larmor precession rate, multiplied by the Landé factor of the excited state, equals the radiative damping rate.

$k_{c}^{(2)}$ is a collisional depolarization factor. The Hanle depolarization depends on the assumed angular distribution of the field vectors, as illustrated for two special cases. From Stenflo (1982)

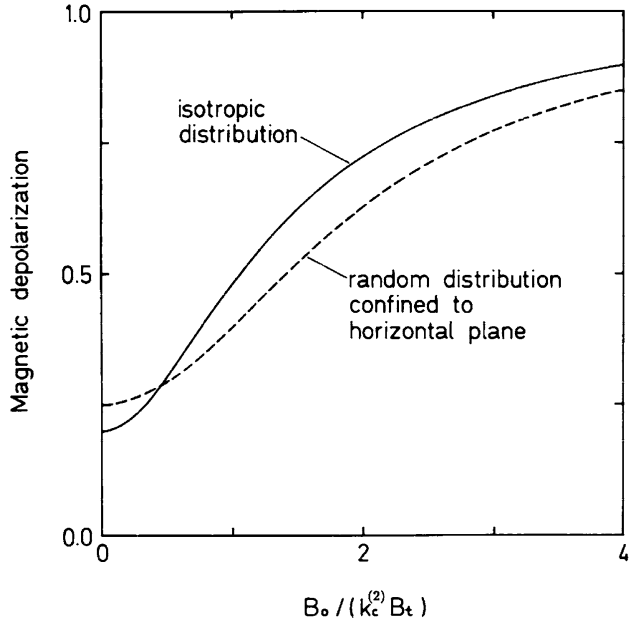

for the optically thin scales in the photosphere. As these scales are much smaller than the atmospheric scale height, we expect the distribution to be close to isotropic.

Realistic ensembles of magnetic flux elements do not have a single field strength but are characterized by a probability density function PDF that describes the field strength distribution. Various such PDFs have been applied to the interpretation of the observed Hanle depolarization (Trujillo Bueno et al. 2004; Stenflo 2012b), and all physically reasonable choices lead to average field strengths that are substantially larger than what is obtained with the single-valued assumption. The observational constraints may be enhanced by the application of the differential Hanle effect (Stenflo et al. 1998), using combinations of spectral lines with different sensitivities to the Hanle effect and combinations of solar regions where the observed depolarizations in the chosen set of lines are found to be different.

\subsection{Forward-scattering Hanle effect}

While the scattering polarization with the Hanle effect is normally best observed close to the solar limb due to favorable scattering geometry (large-angle scattering that resembles the $90^{\circ}$ scattering case), horizontal magnetic fields can generate scattering polarization everywhere on the solar disk, even at disk center (Trujillo Bueno 2001; Trujillo Bueno et al. 2002). This so-called forward-scattering Hanle effect has considerable potential as a diagnostic tool for horizontal magnetic fields in the solar chromosphere.

The principle can be understood by considering for simplicity the idealized case of extreme limb darkening, i.e., when the incident radiation field is assumed to be almost entirely in the vertical direction. Then the transverse plane of the incident radiation is the horizontal plane. Unpolarized incident radiation induces dipole oscillations in the scattering atomic system, which are axially symmetric, i.e., they have a circular distribution when viewed from above, as is done when the center of the solar disk is observed. In the absence of magnetic fields the emitted radiation in the forward direction is therefore unpolarized. 
Fig. 8 Signatures of the forward-scattering Hanle effect in the chromospheric core of the Ca I $4227 \AA$ line in Stokes $Q / I$ and $U / I$. The recording was made near disk center, at $\mu \approx 0.94$, with the ZIMPOL-3 polarimeter at IRSOL (Istituto Ricerche Solari Locarno). In combination with $V / I$ these signatures can be used to diagnose vector magnetic fields in the Sun's chromosphere. From Bianda et al. (2011)

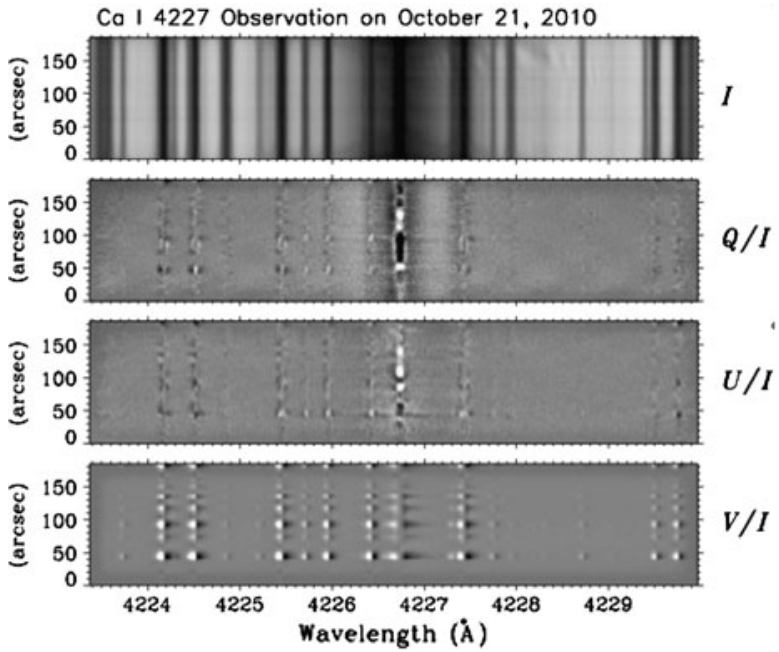

If we now introduce a horizontal magnetic field, the exponentially damped dipole oscillations along the field are unaffected, but the components perpendicular to the field are subject to Larmor precession around the field vector. The result will be a rosette pattern, which when viewed from above has an elliptical shape with the long axis along the field. The emitted radiation will then be linearly polarized along the field direction, and the polarization amplitude will depend on the ratio between the Larmor precession rate and the damping rate as for the large-angle Hanle effect.

The large- and small-angle Hanle effect therefore act in opposite directions. In the case of large-angle scattering the maximum polarization amplitude corresponds to the nonmagnetic case, and the magnetic field causes depolarization and rotation of the plane of polarization. In the case of small-angle scattering the nonmagnetic case is nearly unpolarized, and magnetic fields induce linear polarization along the field direction.

Figure 8, from Bianda et al. (2011), illustrates an example of the forwardscattering Hanle effect in the Ca I $4227 \AA$ line. The Hanle signatures are confined to the Doppler core of this chromospheric line and are very structured along the slit, in different ways for $Q / I$ and $U / I$. They reveal the presence of spatially resolved horizontal magnetic fields in the chromosphere that vary in both magnitude and orientation along the slit. The signatures in the circular polarization $V / I$ are caused by the longitudinal Zeeman effect and represent the line-of-sight component of the field. The $Q / I, U / I$, and $V / I$ signals provide three observational constraints, from which the chromospheric vector magnetic field can be derived. The radiative-transfer theory for the interpretation of the forward-scattering Hanle effect has been developed by Anusha et al. (2011) and applied to interpret the data in Fig. 8.

The forward-scattering Hanle effect is of particular importance for the diagnostics of horizontal magnetic fields in the solar chromosphere because of the absence of good alternatives. Since the chromospheric spectral lines are broad and the chromospheric fields are weak, the transverse Zeeman effect is generally too small to be used. The Hanle effect on the other hand is not diminished by the line width, and it is sensitive to much weaker fields than the Zeeman effect. 


\section{The Sun's global dynamo}

Magnetic fields govern almost all variability in the universe on intermediate time scales. The generation of macroscopic magnetic fields is generally understood in terms of dynamo processes (Larmor 1919; Elsasser 1946, 1956), through induction effects when the highly conductive medium dynamically interacts with a seed field. Dynamo processes are believed to be responsible for the build-up and maintenance of planetary, stellar, interstellar, and intergalactic magnetic fields. The Sun with its $11-y r$ activity cycle represents an oscillatory dynamo with a period of $22 \mathrm{yr}$ (the Hale cycle), since the solar magnetic field reverses sign every 11 years. The Sun is a prototypical dynamo that because of its proximity serves as a unique laboratory, where the dynamo processes can be explored in detail.

The dynamo origin of the global properties of the magnetic-field pattern and its cyclic variation lies in the rotation of the Sun, which through the Coriolis force statistically breaks the left-right symmetry of the convective motions, making them cyclonic with a net helicity (Parker 1955; Steenbeck and Krause 1969). It is the interaction of the magnetic field with the convective or turbulent motions in a highly conductive and rotating medium that causes the global magnetic field of not only the Sun, stars, and planets, but also of galaxies.

This type of dynamo, in which rotation plays a vital, symmetry-breaking role, is generally referred to as the global dynamo, to distinguish it from the local dynamo, which does not need rotation, does not contribute to any stellar activity, and is statistically time invariant. It will be dealt with in the next section.

An early phenomenological description of how the global solar dynamo operates has been provided by Babcock (1961) and Leighton (1969). The frozen-in field lines of an initially poloidal field get wound up by differential rotation. This leads to the generation and amplification of a toroidal field. Buoyancy forces cause sections of subsurface toroidal flux ropes to be brought to the Sun's surface. The field breaks through the surface in the form of bipolar magnetic regions, which may contain sunspots where the flux concentration is sufficiently large to inhibit convective energy transport from the Sun's interior.

When Coriolis forces act on the buoyantly rising flux ropes, they acquire a systematic tilt with respect to the $\mathrm{E}-\mathrm{W}$ direction. The tilt leads to an $\mathrm{N}-\mathrm{S}$ bipolar moment that serves as the seed for the new poloidal field with reversed polarity. The magnetic field from all the emerged bipolar fields of all sizes spread by turbulent diffusion to replace the old global poloidal field with a new one having opposite orientation.

The dynamo of the real Sun is much more stochastic and fluctuating than the Babcock-Leighton scenario with its coherent subsurface flux ropes suggests. The rotation is differential both in latitude and depth, meridional circulations play a role, and the main location of the dynamo action is still under debate (tachocline dynamo at the bottom of the convection zone, or distributed dynamo throughout the convection zone). For more details, we refer to various reviews (e.g. Brandenburg and Subramanian 2005; Brandenburg 2005; Charbonneau 2010).

\subsection{Hale's polarity law}

The full-disk magnetogram in Fig. 9 illustrates one of the fundamental properties of the magnetic pattern produced by the global solar dynamo: the anti-symmetric E-W 
Fig. 9 Full-disk magnetogram recorded by the HMI instrument on the SDO spacecraft (Scherrer et al. 2012; Schou et al. 2012) on January 4, 2013. The map, representing the line-of-sight component of the magnetic flux density, is based on recordings of the circular polarization in the Fe I $6173 \AA$ line with a spatial resolution of 1 arcsec. Notice that there is not only one latitude zone of activity in each hemisphere but a coexistence of several distinctly different latitude zones. Courtesy of NASA/SDO and the AIA, EVE, and HMI science teams

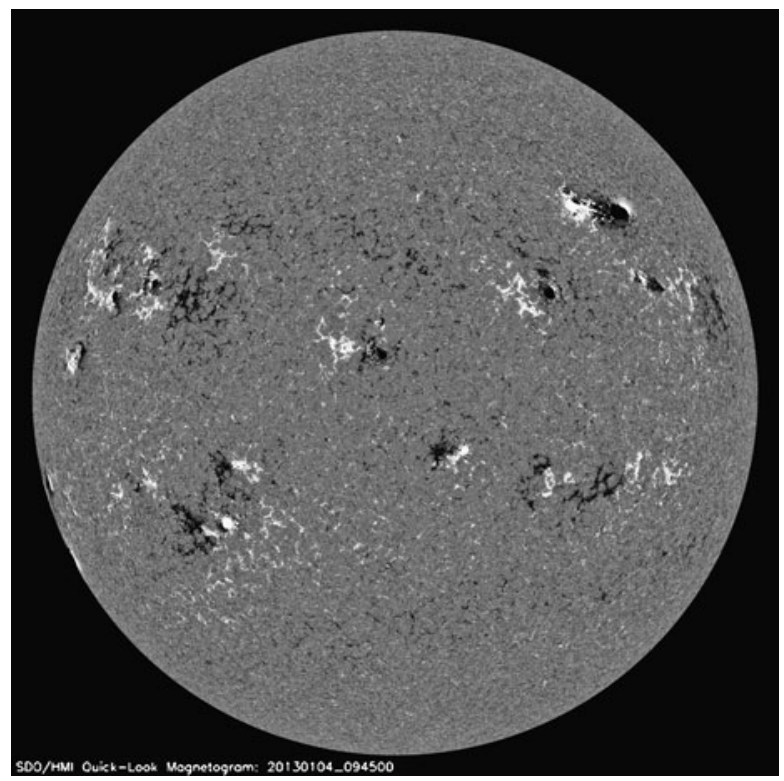

polarity orientation of the bipolar magnetic regions. In the figure, heliographic $\mathrm{N}$ is up, E to the left. Brighter regions represent positive polarity (field directed towards us), darker regions negative polarity. We notice that in the northern hemisphere the E-W polarity orientation is +- (bright to the left of dark), while in the southern hemisphere it is the opposite $(-+)$.

This anti-symmetry with respect to the solar equator can be understood in terms of the systematic winding up of the poloidal field by the differential rotation. In the Babcock-Leighton scenario the toroidal field is first amplified at higher latitudes, where the first sunspots of the new cycle appear, after which the activity zone gradually migrates to lower latitudes during the course of the cycle. This latitude migration is generally represented as isocontours in latitude-time space ("butterfly diagrams").

According to this scenario we would at any given time expect to have one distinct activity belt of bipolar magnetic regions in each hemisphere, which migrates with the phase of the cycle. Note however that this is not at all the case for the pattern illustrated in Fig. 9, which reveals the coexistence of several toroidal flux belts in each hemisphere. The high- and low-latitude bipolar regions in the northern hemisphere have the same polarity orientation, implying that they belong to the same activity cycle.

The onset of a new 11-yr activity cycle is marked by the appearance of highlatitude bipolar regions with the reversed polarity orientation, followed by a new migration towards the equator. Two cycles may overlap, with a coexistence of lowand high-latitude bipolar regions with opposite polarity orientations. Note, however, that the pattern seen in Fig. 9 does not represent a case of overlapping cycles.

The property of the magnetic pattern that the E-W polarity orientation of the bipolar magnetic regions is anti-symmetric with respect to the equator and reverses sign with each new 11-yr cycle is called Hale's polarity law (Hale et al. 1919). The full magnetic cycle is therefore $2 \times 11 \mathrm{yr}$, the 22-yr Hale cycle. 
Fig. 10 Examples of violations of Hale's polarity law by medium or large-size bipolar magnetic regions from different phases of the solar cycle between 2004 and 2010, from Stenflo and Kosovichev (2012). The violating regions with reversed orientations are marked by the rectangular boxes, the solar equator by the dashed lines. In all cases there are bipolar regions with the correct orientation in the same latitude zone of the same magnetogram, which is evidence that they cannot originate from a common subsurface toroidal flux system
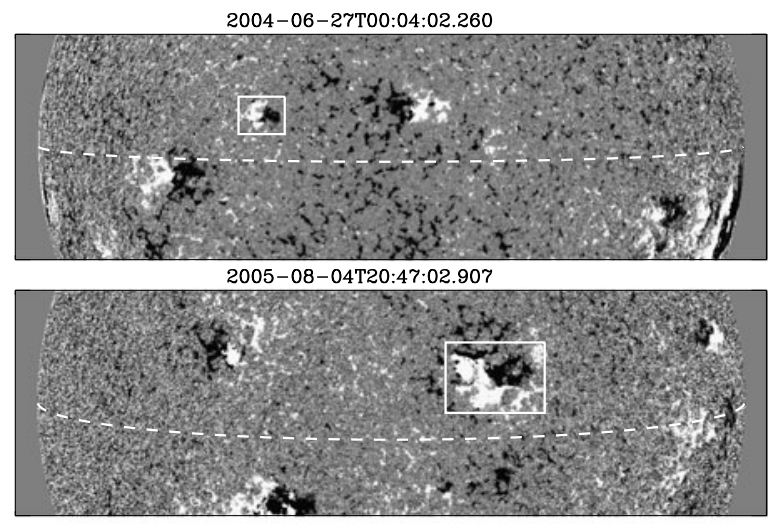

2008-01-11T07:59:01.789

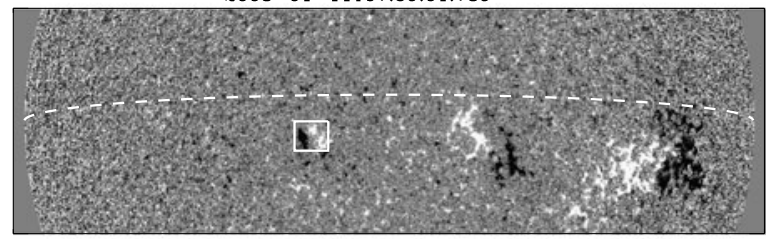

2010-02-16T09:39:02.339

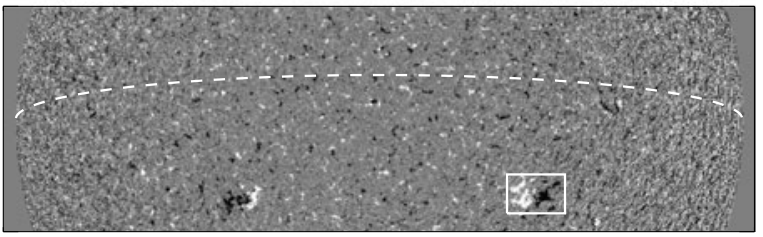

In the Babcock-Leighton scenario with coherent subsurface flux ropes wound up by the Sun's differential rotation, one would expect strict obedience to Hale's polarity law. There are however numerous violations of this law. In Fig. 10, from Stenflo and Kosovichev (2012), four such violations from different phases of the solar cycle are illustrated. They represent cases where medium or large-size bipolar regions with opposite E-W polarity orientations occur side by side in the same magnetogram. They clearly cannot be part of the same subsurface flux rope, but reveal the coexistence of toroidal subsurface flux systems with opposite orientations at the same latitudes, something that cannot be accounted for in the Babcock-Leighton scenario.

The time sequences of magnetograms have been inspected to verify that the violating regions do not exhibit any significant rotation but represent stable polarity orientations. For medium to large-size bipolar regions Stenflo and Kosovichev (2012) find that typically $4 \%$ (possibly less for the largest regions) violate Hale's law. This is consistent with the conclusions of Richardson (1948), Wang and Sheeley (1989), Khlystova and Sokoloff (2009), and Sokoloff and Khlystova (2009). As we go to smaller bipolar regions, however, the fraction of violating regions is found to increase dramatically, which indicates that the polarity orientations get randomized in the small-scale limit.

The violations of Hale's law are not only in the form of reversed E-W orientation, but there is also a similar frequency of regions with $\mathrm{N}-\mathrm{S}$ polarity orientation (Stenflo 
Fig. 11 Latitude variation of the average tilt angles of bipolar magnetic regions, expressing Joy's law. Positive tilt means that the preceding polarity is equatorwards of the following polarity. The solid line is the analytical fit function $32.1^{\circ}$ $\sin$ (latitude). From Stenflo and Kosovichev (2012)

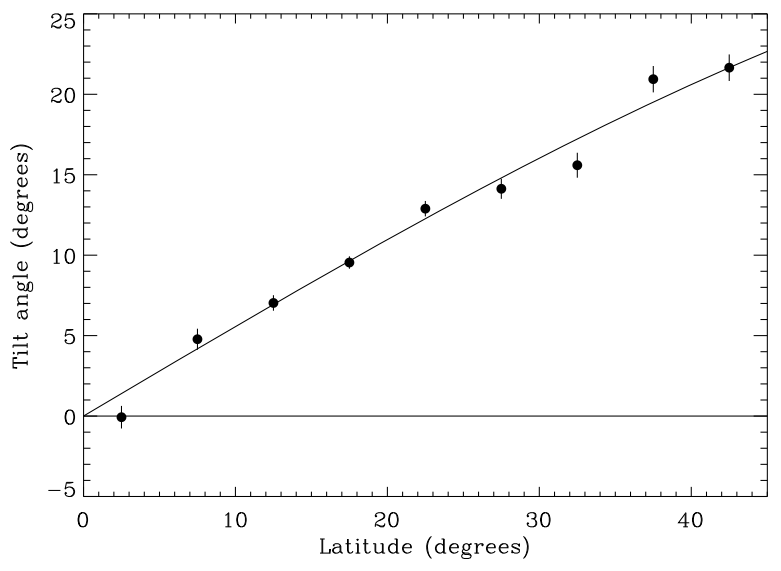

and Kosovichev 2012), which should not occur for an origin in terms of coherent toroidal flux ropes. This shows that the simplistic phenomenological model of coherent flux ropes needs to be replaced by a dynamo scenario in which fluctuations on all scales play a major role.

\subsection{Joy's law}

Another fundamental law discovered through the pioneering Zeeman-effect work of Hale and collaborators (Hale et al. 1919) is Joy's law that governs the tilt angles of bipolar magnetic regions. Although the main orientation is in the $\mathrm{E}-\mathrm{W}$ direction, there is a systematic tilt with respect to this direction, such that the preceding (westward) part of the region is systematically closer to the equator than the following part. It is the average tilt angle and its latitude variation that is governed by Joy's law.

While Hale's polarity law expresses how a toroidal magnetic field is generated from a poloidal field, Joy's law embodies the generation of a poloidal magnetic field from a toroidal one, because the tilt implies an emerged $\mathrm{N}-\mathrm{S}$ bipolar moment that serves as a seed for the new large-scale poloidal field. Since the following polarity of the bipolar regions is opposite to the polarity of the polar field and spreads by turbulent diffusion more towards the pole than the preceding polarity, it will eventually cause polar-field reversal and the creation of a global poloidal field with reversed orientation every 11 years.

The tilt angles of the bipolar regions have a large intrinsic scatter due to the fluctuating nature of the solar dynamo, so an accurate determination of Joy's law has only recently become possible with massive statistics. Through analysis of the 73838 magnetograms of the entire SOHO/MDI data set with a computer algorithm that automatically identifies bipolar magnetic regions of various sizes, the precise latitude variation of the average tilt angle could be determined. The result is illustrated in Fig. 11, from Stenflo and Kosovichev (2012). The empirically determined tilt angles can be closely represented by the simple analytical function $32.1^{\circ} \sin$ (latitude). The main source of the systematic tilt angles is believed to be the Coriolis force, which also varies with the sine of the latitude. 
The rather large tilt angles implied by Fig. 11 are in good general agreement with the results of a number of other studies (Wang and Sheeley 1989; Li and Ulrich 2012; Tlatov et al. 2013), but disagree with the much flatter latitude dependence that was found from Mount Wilson and Kodaikanal data by Dasi-Espuig et al. (2010) and derived from numerical simulations by Schüssler and Baumann (2006). Li and Ulrich (2012) searched for temporal variations over the period 1974-2012, which spans nearly four solar cycles, and found the tilt angle for each latitude zone to be time invariant.

There is no indication that Joy's law as expressed by Fig. 11 has any significant dependence on region size. According to the study of Stenflo and Kosovichev (2012), bipolar regions differing as much as four orders of magnitude in their flux content seem to follow the same law. Although the average tilt (for a given latitude zone) seems to be the same, the scatter of the tilt values increases dramatically as we go to smaller regions. It should, however, be mentioned that Tlatov et al. (2013), while agreeing with the results of Stenflo and Kosovichev (2012) for the larger bipolar regions, claim that small, ephemeral-type bipolar regions have similar magnitudes for the tilt angles but reversed orientations with respect to the large regions. This intriguing claim is difficult to understand and needs to be verified.

\subsection{Scales that feed the oscillatory dynamo}

Bipolar magnetic regions occur on all scales and have a size spectrum that follows a power law (Harvey 1993; Harvey and Zwaan 1993; Schrijver and Harvey 1994; Parnell et al. 2009), from the largest regions that harbor major sunspots to ephemeral active regions (Harvey and Martin 1973; Harvey et al. 1975; Martin and Harvey 1979) without sunspots, and the still smaller internetwork fields. The global contribution to the overall flux emergence rate dramatically increases as we go down in scale size (Zirin 1987), suggesting that the global flux balance could be dominated by the smallest scales. Let us therefore next try to determine which scales contribute the most to the regeneration of the poloidal field from the toroidal one.

Harvey (1993) found from analysis of Kitt Peak full-disk magnetograms that the emergence rate $R$ of bipolar magnetic regions has a smooth and continuous scale dependence. Within the error bars the histogram distribution $\mathrm{d} R / \mathrm{d} A$ as a function of region area $A$ may be described in terms of the power law

$$
\mathrm{d} R / \mathrm{d} A \sim A^{-p}
$$

with a power law index of $p \approx 3$.

Using a feature recognition algorithm Parnell et al. (2009) analyzed magnetograms recorded by SOHO/MDI and by Hinode/SOT to determine the size distribution of solar magnetic structures. They found that the distribution follows a power law over 5 orders of magnitude in flux $\Phi$. If $N$ is the number of magnetic features at a given time,

$$
\mathrm{d} N / \mathrm{d} \Phi \sim \Phi^{-\alpha}
$$

with a power law index of $\alpha=1.85 \pm 0.14$. 
Let us now examine how these two relations are related to each other. Each structure has an average life time $\tau$ before it dissolves, most likely through fragmentation, cascading to higher wave numbers in the magnetic energy spectrum. Various studies of the life time $\tau$ of solar magnetic structures indicate that $\tau \sim A \sim d^{2}$, where $d$ is the size of the structure (cf. Stenflo 1976; Harvey 1993). Such a size dependence of the life time is expected if the evolution of the structure obeys a diffusion equation (fragmentation through the interchange instability followed by turbulent diffusion). Since the emergence rate $R$ is related to the number of features through $N=R \tau$, it follows that

$$
\mathrm{d} N / \mathrm{d} A \sim A^{-p+1} .
$$

The magnetic flux of a magnetic element can be written as $\Phi=B A=B d^{2}$, where $B$ is the field strength. Most of the flux that is visible in magnetograms is in collapsed $\mathrm{kG}$ form and the dependence of $B$ on size $d$ is generally weak. If we disregard this dependence, then $\Phi \sim A$, and we retrieve Eq. (20) from Eq. (21) if $p=\alpha+1$. With $\alpha=1.85$ we then get $p=2.85$, which agrees within the uncertainties with the value of 3 estimated from the data of Harvey (1993).

Of importance for the regeneration of the poloidal field is not only the amount of emerged flux $F$, but also the separation $S$ between the opposite polarities. The key parameter is the bipolar moment, defined in Stenflo and Kosovichev (2012) as $M=\frac{1}{2} F_{\text {tot }} S$, where $F_{\text {tot }}$ is the sum of the unsigned positive and negative magnetic flux of the bipolar region. The $\mathrm{N}-\mathrm{S}$ component of the bipolar moment is then obtained through multiplication of $M$ with the sine of the tilt angle (which appears to be independent of region size).

Since $S$ scales with the size $d$ of the structures, $M \sim B d^{3}$. Let us by $R_{\mathrm{NS} \text {-moment }}=$ $M N / \tau$ denote the emergence rate of the $\mathrm{N}-\mathrm{S}$ bipolar moment. If we disregard the weak $d$-dependence of $B$ and the average tilt angle, and make use of Eq. (21) and $\tau \sim d^{2}$, we get

$$
R_{\text {NS-moment }} \sim d^{-2 p+5} .
$$

With our previous value $p=3$ from Harvey (1993) we find $R_{\mathrm{NS} \text {-moment }} \sim 1 / d$, which implies that it is the smallest scales that contribute the most to the regeneration of the poloidal field. The rate diverges in the small-scale limit unless there is a cut-off mechanism. Such a mechanism is provided by the randomization of the tilt angles.

In the analysis of Stenflo and Kosovichev (2012) the histograms of tilt angles for each latitude zone were fit with an analytical model that represented a decomposition with two components, one oriented component with a Gaussian tilt angle distribution, and one flat distribution representing random tilt angles. The average tilt angle for the Gaussian component is what has been plotted in Fig. 11 to represent Joy's law. The random component does not contribute to or affect this average, but it determines the fraction of the emerged flux that contributes to the regeneration of the poloidal field.

If we denote the relative occurrence of the oriented and random components (integrated over all tilt angles) by $f_{\text {oriented }}$ and $f_{\text {random, }}$, then it is the fraction $r=$ $f_{\text {oriented }} /\left(f_{\text {oriented }}+f_{\text {random }}\right)$ that contributes to the poloidal field regeneration. Based on the data from the analysis of Stenflo and Kosovichev (2012) we have in Fig. 12 plotted this fraction as a function of bipolar moment. We find that it has a rather sharp 
Fig. 12 Relative fraction of bipolar magnetic regions of various sizes that may contribute to the global N-S bipolar moment, which regenerates the Sun's poloidal magnetic field. The cut-off range of $10-100 \times 10^{20} \mathrm{Mx} \mathrm{Mm}$ corresponds to an approximate size range of 2-8 $\mathrm{Mm}$. The data points (filled circles and dashed line) have been extracted from the analysis material of Stenflo and Kosovichev (2012), while the solid line is an analytical fit curve

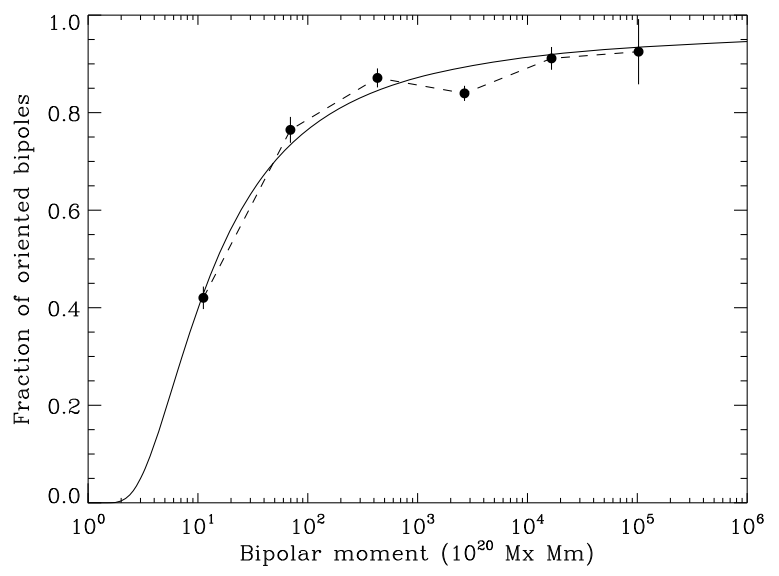

cut-off near a bipolar moment of $M \approx 10-100 \times 10^{20} \mathrm{Mx} \mathrm{Mm}$, below which there is no significant contribution to the $\mathrm{N}-\mathrm{S}$ bipolar moment.

Since we may write $M=m B d^{3}$, where $m$ is a dimensionless number of order $2-10$, depending on how the polarity separation scales with size $d$ of each of the two main polarity structures, we can estimate the scale size $d$ to which a given $M$ corresponds. With our generous allowed range for parameter $m$ and assuming $B=$ $1 \mathrm{kG}$, we find that the cut-off of Fig. 12 corresponds to scales of order $d=2-8 \mathrm{Mm}$.

According to Eq. (22) it is the smallest scales that contribute the most to the regeneration of the poloidal field through the emergence of bipolar regions, as long as we are above the cut-off. From our previous estimates it then follows that bipolar magnetic regions with sizes of order $10 \mathrm{Mm}$ appear to be the main contributors.

This result might seem to contradict claims that the largest bipolar regions provide sufficient flux to explain the surface field evolution in terms of the BabcockLeighton flux transport model of the solar cycle (e.g. Sheeley et al. 1985; Jiang et al. 2013). However, since these models are idealized and contain adjustable parameters, and since the large bipolar regions and the accumulated contributions from the small bipoles affect the surface pattern in qualitatively similar ways, the flux transport models cannot be used as evidence against a dominant role of the smaller bipoles. By adjusting the models to compensate for the neglected small-scale contributions, the impression may arise that these contributions do not play a significant role.

\section{Local dynamo}

The global dynamo is responsible for all of solar activity, with sunspots, flares, CMEs, etc. The cyclic variation has its origin in the left-right symmetry breaking of the turbulent motions induced by the Sun's rotation via the Coriolis force. The word "global" does not imply "large-scale". The dynamo action takes place on all scales. The generated magnetic fields at the larger scales cascade down the magnetic energy spectrum until they reach the magnetic diffusion limit around a scale of order $25 \mathrm{~m}$, where the magnetic Reynolds number becomes unity and the field lines decouple 
Fig. 13 Illustration of the nearly perfect correlation between the disk average of the unsigned vertical magnetic flux density derived from SOHO/MDI full-disk magnetograms (solid line) and a second-order polynomial fit of the relative sunspot number $R_{z}$ (dashed line). It shows that the average basal flux density of the Sun, in the absence of sunspots, is $2.7 \mathrm{G}$ for the 4 arcsec scale of the MDI recordings. From Stenflo (2012a)

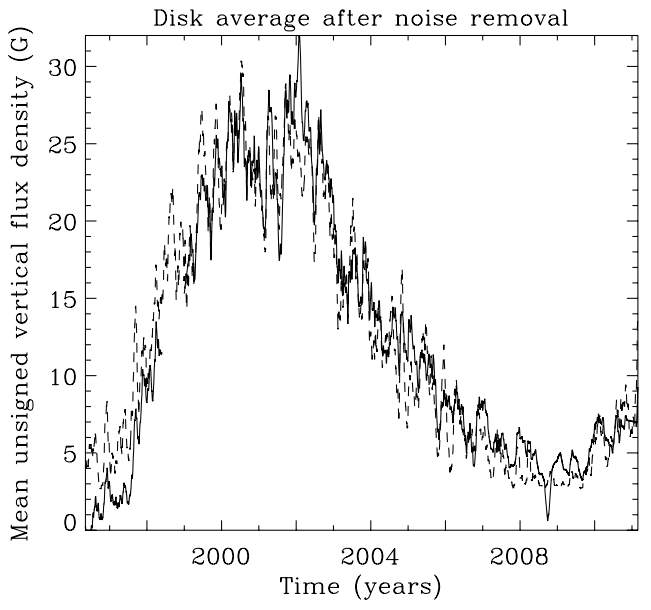

from the plasma, because the frozen-in condition is not valid for smaller scales (cf. Stenflo 2012b). The global dynamo is therefore a source of structuring throughout the entire scale spectrum. It is not meaningful to separate the global dynamo into a small-scale and a large-scale part, all scales are connected.

Based on theoretical considerations and numerical simulations it has, however, been suggested that there exists a second, qualitatively different dynamo that also operates on the Sun and is responsible for the statistically time-invariant small-scale structuring of the magnetic field (Petrovay and Szakaly 1993; Cattaneo 1999; Vögler and Schüssler 2007).

The circumstance that the magnetic field is highly structured on small scales, far below the resolution limit of current telescopes, is sometimes incorrectly taken as evidence for a local dynamo. Small-scale structuring is also produced by the global dynamo via the turbulent cascade. What distinguishes the two dynamos from each other is that the local dynamo is decoupled from solar activity and contributes to a background quiet-sun magnetic pattern that is statistically constant over the Sun and does not vary with the cycle. We call such magnetic flux basal flux.

Analysis of the full SOHO/MDI data set of full-disk magnetograms shows that there is an almost perfect correlation between the disk average of the unsigned vertical magnetic flux density and the sunspot number $R_{z}$ (Stenflo 2012a), as illustrated in Fig. 13. In the absence of sunspots the MDI basal flux density is found to be $2.7 \mathrm{G}$, which represents an upper limit to the possible contributions from a local dynamo at this spatial scale (4 arcsec). Much of this apparently basal flux may well originate from the global dynamo, since the background flux pattern that has been generated by the breakup and turbulent diffusion of bipolar magnetic regions will not suddenly disappear when solar activity is switched off. Much of the background pattern may be expected to survive throughout the cycle minimum until the new cycle begins to supply new flux from the Sun's interior.

The average unsigned vertical flux density $B_{\text {ave }}$ depends on scale size $d$ according to the cancelation function

$$
B_{\text {ave }} \sim d^{-\kappa}
$$


Fig. 14 Comparison of two SDO/HMI magnetograms of the quiet Sun at disk center ( $x$ and $y$ are the coordinates relative to disk center), recorded at times 2011.2086 (upper panel) and 2011.4796 (lower panel). The gray-scale cuts are the same for both panels $( \pm 50 \mathrm{G})$. The top panel represents a region with the basal flux density level (3 G) for the 1 arcsec scale of HMI, while the bottom panel shows a region with 2.2 times more flux. From Stenflo (2012a)

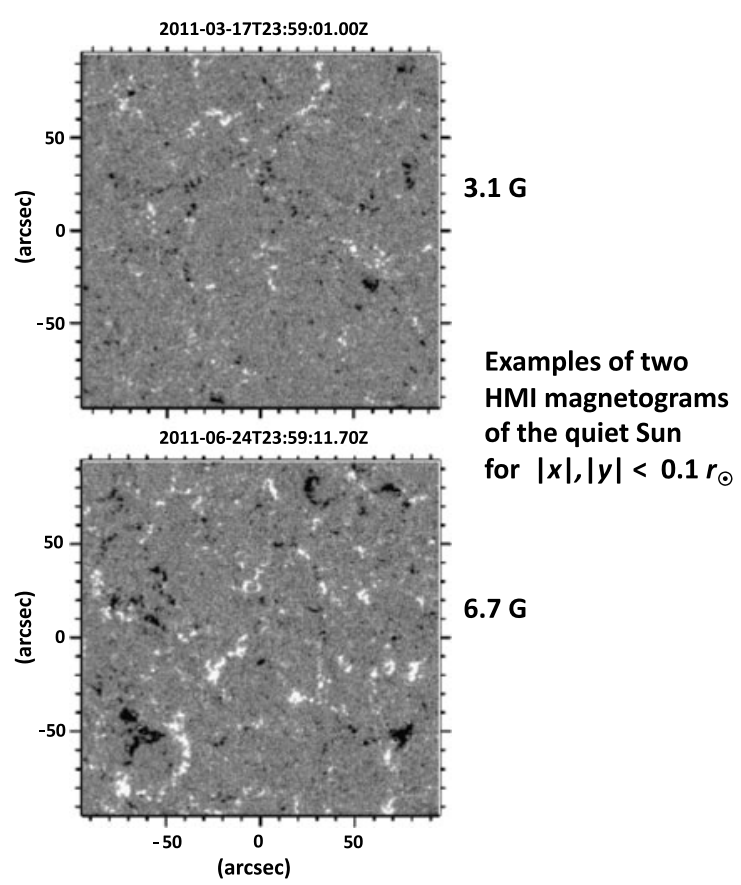

as determined from Hinode SOT/SP data for a scale range from the Hinode resolution scale $(230 \mathrm{~km})$ up to and beyond the MDI scale (4 arcsec) (Pietarila Graham et al. 2009; Stenflo 2011). Consistent results are obtained with a cancelation exponent $\kappa=0.13$. Analysis of magnetograms obtained with SDO/HMI during the deep cycle minimum 2010-2011 gives a basal vertical flux density at disk center of $3.0 \mathrm{G}$ for the HMI spatial scale (1 arcsec), which is consistent with the MDI value of $2.7 \mathrm{G}$ and the scaling law of Eq. (23) with $\kappa=0.13$. Extrapolated with this law to the Hinode spatial scale, the basal flux density is $3.5 \mathrm{G}$ (Stenflo 2012a).

The average flux density at the quiet-sun disk center varies greatly with time, as illustrated in Fig. 14. Such variations have nothing to do with a local dynamo, which must be statistically constant. Instead they indicate that much, possibly all, of the flux that we see on the quiet Sun is supplied by the global dynamo. The magnetic pattern in the upper panel of Fig. 14, which is representative of the basal flux density, is qualitatively similar to the pattern in the bottom panel, which represents 2.2 times more flux. Both patterns exhibit network-like structuring on the supergranular scale, the difference lies only in the relative amounts of available flux.

Since the upper limit to the possible contribution from a local dynamo to magnetic structuring at the Hinode scale is as low as $3.5 \mathrm{G}$, while the average unsigned flux densities that have been reported from Hinode SOT/SP observations of the quiet-sun disk center are typically three times larger (Lites et al. 2008; Stenflo 2010a), it is clear that most, if not all, of the magnetic structuring revealed by Hinode on the quiet Sun has its origin in the global dynamo, not in a local dynamo.

It should be mentioned that this conclusion is still controversial, in particular because of claims that the ubiquitous and statistically time-invariant horizontal mag- 


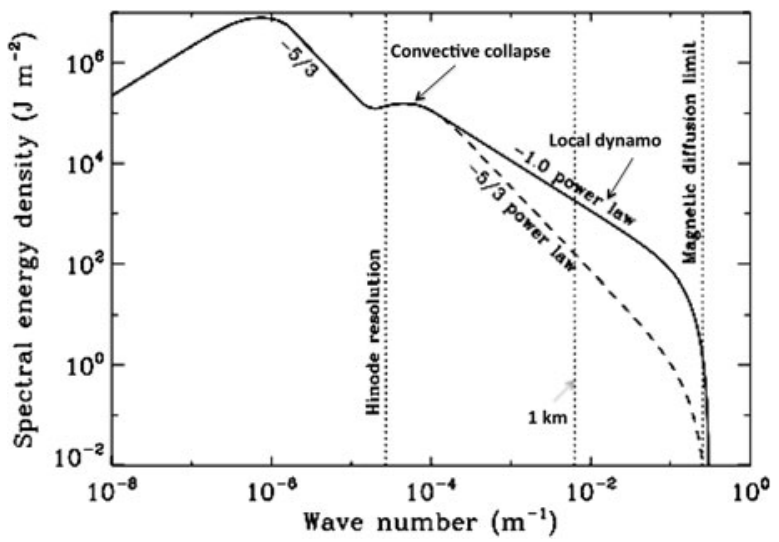

Fig. 15 Magnetic energy spectrum of the solar photosphere, spanning seven orders of magnitude in wave number, adapted from Stenflo (2012b). A -5/3 Kolmogorov power law in the spatially unresolved domain would not give rise to sufficient magnetic structuring at small scales to account for the observed Hanle depolarization. If the spectrum is raised to follow a -1.0 power law instead, it becomes consistent with the Hanle constraint. As so much power at small scales is inconsistent with the scaling behavior at the resolved scales, which are governed by the global dynamo, it is conjectured that a local dynamo is operating as an additional source of magnetic flux at scales below about $10 \mathrm{~km}$

netic fields inferred from linear-polarization measurements with Hinode give evidence for a local dynamo at the Hinode spatial scale (e.g. Buehler et al. 2013). However, as indicated by Fig. 5, the Hinode linear polarization recorded on the quiet Sun is dominated by noise, and the instrumental noise is time invariant. As shown in Stenflo (2013) and described in Sect. 9.1 below, the photospheric magnetic fields at the center of the quiet solar disk have a preferentially vertical, not horizontal, angular distribution, contrary to repeated claims from analysis of Hinode data. This conclusion is both resolution and model independent, as will be explained in Sect. 9.1.

Although there may not be a significant role of a local dynamo at any of the resolved spatial scales, it is likely that the local dynamo plays a dominating role at scales below about $10 \mathrm{~km}$. The reason is that the vast amounts of "hidden" magnetic flux that have been revealed by the observed Hanle effect depolarization (cf. Sects. 4.3 and 9.2) cannot be explained only in terms of the turbulent cascade of flux generated by the global dynamo, an additional source of flux is needed.

Figure 15 gives an overview of the magnetic energy spectrum of the quiet Sun. The part to the left of the vertical dotted line (the Hinode resolution limit at $230 \mathrm{~km}$ ) has been directly determined from the observations, since it represents the spatially resolved domain. The bump in the scale range of about 10-100 km has been inferred from Hinode Stokes $V$ line-ratio data and represents the population of kG-type fields that have been spontaneously generated by the convective collapse instability. As we go to higher wave numbers the structures get much smaller than the atmospheric scale height and therefore experience an increasingly isotropic environment. According to Kolmogorov (1941) theory one would then expect a $-5 / 3$ power law for the turbulent cascade in this inertial range, which ends at the magnetic diffusion limit (here estimated to be at the $25 \mathrm{~m}$ scale), where the magnetic Reynolds number becomes unity and the magnetic field lines cease to be frozen-in. 
The observed Hanle depolarization (cf. Sects. 4.3 and 9.2) implies the existence of a vast hidden ocean of tangled or turbulent fields (Stenflo 1982) with an average unsigned flux density of at least $60 \mathrm{G}$ (Trujillo Bueno et al. 2004). It is largely invisible in magnetograms due to cancelation of the contributions from the opposite polarities that are mixed on subresolution scales. The scaling law represented by the cancelation function of Eq. (23), which was empirically determined in the resolved regime that is dominated by the global dynamo, is much too shallow to provide anything close to the flux density that is needed when one extrapolates it down to the magnetic diffusion limit. Similarly the $-5 / 3$ power law of the magnetic energy spectrum is much too steep to provide sufficient magnetic structuring at small scales needed to satisfy the Hanle constraint. To restore consistency with the Hanle observations we find it necessary to raise the energy spectrum to the level of a -1.0 power law in the small-scale inertial range. The cancelation function that is valid in the resolved domain then cannot be expected to retain its validity at the smallest scales, since the flux there is supplied by a different mechanism, which is insignificant at resolved scales.

\section{Global evolution}

The appearance of the corona during a total solar eclipse indicates that the Sun is a magnetized sphere that possesses a global, dipole-like magnetic field. Soon after his discovery of magnetic fields in sunspots (Hale 1908), George Ellery Hale and his team began to apply the Zeeman effect to determine the dipole-like structure of the general magnetic field. In a series of papers (e.g. Hale et al. 1918) they reported successful results. Many of Hale's assistants did independent visual analysis of the many photographic plates of the Zeeman effect. About half of them obtained null results, but Hale's conclusions were largely based on one of his assistants, Van Maanen, who consistently got well-defined positive results with small error bars.

These results later turned out to be entirely inconsistent with modern photoelectric measurements (made possible with the Babcock 1953 magnetograph), which revealed a general magnetic field weaker by an order of magnitude and with a polarity that according to the Hale cycle should have been opposite to the one found by Hale and Van Maanen at the time of their measurements. For this reason a batch of about 400 photographic plates analysed by Van Maanen in 1914 were located and remeasured with a modern digitized microphotometer (Stenflo 1970). While Van Maanen, using the identical plate material, found a strong dipole-like field with a well-defined latitude variation that was anti-symmetric around the equator, the modern reanalysis gave null results with error bars that ruled out his results, which can only be understood as the result of severe subjective bias.

Van Maanen also measured the proper motions of the stars on photographic plates of the spiral nebula M33. The large proper motions that he found could only be understood if M33 were a nearby object. They were therefore seen as strong evidence against the extragalactic nature of spiral nebulae. We now know this result to be wrong, another example of subjective bias (cf. Stenflo 1970).

Full-disk solar magnetograms have been recorded on a daily basis, starting at the Mt Wilson Observatory in August 1959, with higher spatial resolution at the National 
Solar Observatory/Kitt Peak since December 1976, with the SOHO/MDI magnetograph in space May 1996-April 2011, and presently continued by SDO/HMI with 1 arcsec resolution and $45 \mathrm{~s}$ cadence. A convenient overview of the evolutionary information contained in the sequence of full-disk magnetograms is provided by synoptic maps, showing the distribution of the line-of-sight component of the magnetic field as a function of latitude and longitude over the whole surface of the Sun. The different longitudes are covered by sampling the longitude strip around the central meridian in each magnetogram. During the course of one full solar rotation, all longitudes get covered.

While the solar plasma rotates differentially, with the period of rotation varying with latitude (and depth), but coordinate systems rotate rigidly, the definition of the solar longitude system is chosen by convention. The standard choice is the Carrington system, which has a period of 27.2753 days. This is the time window covered by a synoptic map. Evolutionary effects over this time scale are mixed with longitude variations in the synoptic maps, since we cannot view the whole range of longitudes simultaneously.

Because the observations only give us the line-of-sight component of the magnetic flux density, the polar fields have poor visibility since they are always located near the solar limb. A physically more relevant representation is in terms of the vertical flux density, but since it is not directly measured, it has to be obtained through projection. This is done by assuming that on average the field is vertical at the atmospheric level where it is measured. The vertical flux density is then obtained from the line-of-sight flux density simply through division by $\mu$, the cosine of the heliocentric angle.

The vertical assumption gets its justification from the observation (through application of the Stokes $V$ line-ratio technique, cf. Sect. 8.2) that more than $90 \%$ of the line-of-sight magnetic flux in magnetograms with moderate or low spatial resolution has its origin in strong, kG-type intermittent flux bundles (Howard and Stenflo 1972; Frazier and Stenflo 1972; Stenflo 1973), usually referred to as flux tubes. These flux tubes are anchored in the subphotospheric layers and get forced towards an upright orientation in the photosphere because they are highly buoyant.

The solar magnetic cycle represents a global oscillation between a toroidal and a poloidal field. The dominating features of solar magnetograms are the bipolar regions, which represent sections of the subsurface toroidal field that have buoyantly risen to protrude through the solar surface, where we can observe them. The poloidal field is less conspicuous and much in the form of a large-scale background pattern. To make the global evolution of the poloidal field more visible one can extract its axisymmetric component by averaging each synoptic magnetic map over all longitudes to obtain the latitude variation of this average as a function of time. Such a representation is illustrated in Fig. 16, which represents the vertical component of the axisymmetric field (obtained from the line-of-sight field via the vertical assumption). A diagram that represents the sunspot number in latitude-time space is called a butterfly diagram, but this name is often used for the magnetic-field representation as well.

The low-latitude belts that migrate towards the equator in Fig. 16 coincide with the activity belts with sunspots. The polarity pattern is anti-symmetric with respect to the equator and reverses each $11 \mathrm{yr}$, as required by Hale's polarity law, although the phase is latitude dependent. Each low-latitude belt is dominated by one polarity, the 


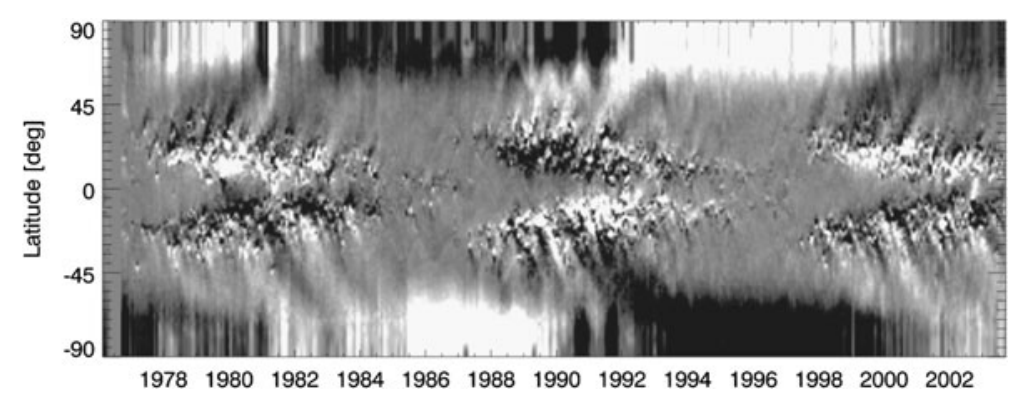

Fig. 16 "Butterfly diagram" of the axisymmetric component of the Sun's radial magnetic field, based on synoptic magnetic maps recorded at NSO/Kitt Peak. Bright regions indicate fields directed out of the Sun, dark regions fields directed towards the Sun. From Schüssler and Baumann (2006)

preceding polarity of the bipolar magnetic regions. Discrete pulses of the opposite (following) polarity shoot out towards the polar regions from the poleward sides of the belts. They supply the polar regions with the new flux that replaces and reverses the old polar field. During the period of minimum solar activity the polar fields reach their maximum amplitude and the global pattern resembles that of a dipole.

\subsection{Harmonic decomposition}

The natural mathematical description of a pattern on a spherical surface is in terms of spherical harmonics. They are used in the formulation of the dynamo equations as an eigenvalue problem (e.g. Steenbeck and Krause 1969). To bring the observational data into a form that is suited for interpretation in terms of dynamo theory, the observed pattern of vertical magnetic fields on the Sun can be decomposed in its spherical harmonics. The global evolution of the field can then be explored in terms of time series analysis of the various harmonic modes (Altschuler et al. 1974; Stenflo and Vogel 1986; Stenflo and Guedel 1988; Knaack and Stenflo 2005; DeRosa et al. 2012).

While such time series analysis reveals a number of intermittent, quasi-periodic variations, in particular biennial oscillations in the period range 1.2-2.5 yr (cf. Knaack and Stenflo 2005), the truly resonant $22 \mathrm{yr}$ oscillation dominates the odd, axisymmetric modes (which represent patterns that are anti-symmetric with respect to the equator). In contrast, this resonance is nearly absent in the even (symmetric) modes.

Let $B(x, \varphi, t)$ be the vertical flux density as a function of $x=\cos \theta$ (where $\theta$ is the colatitude), longitude $\varphi$, and time $t$. Then the axisymmetric field is

$$
\bar{B}(x, t)=\frac{1}{2 \pi} \int_{-\pi}^{\pi} B(x, \varphi, t) \mathrm{d} \varphi .
$$

It can be expanded in terms of the Legendre polynomials $P_{\ell}(x)$ :

$$
\bar{B}(x, t)=\sum_{\ell=0}^{\infty} b_{\ell}(t) P_{\ell}(x) .
$$




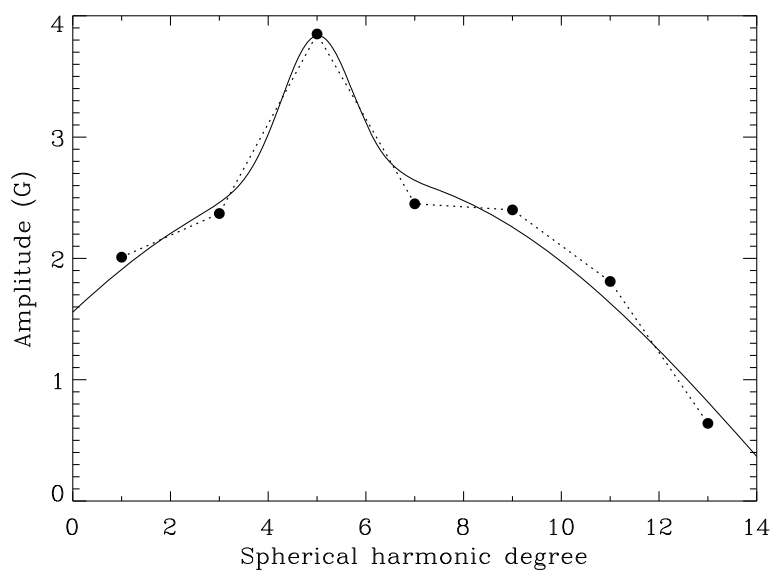

Fig. 17 Amplitude of the 22 yr periodic variations of the axisymmetric modes of the Sun's vertical magnetic-field pattern as a function of spherical harmonic degree $\ell$, as determined from harmonic decomposition of a $33 \mathrm{yr}$ data base compiled from Mt Wilson and Kitt Peak synoptic magnetic-field maps for the period 1959.6-1992.8 (filled circles and dotted line). The solid line represents an analytical fit function to get a smoother representation. Note that the dominating contributor to the axisymmetric pattern is the mode with $\ell=5$. Adapted from Stenflo (1994a)

Using the orthonormality relations for the Legendre polynomials we can invert Eq. (25) to obtain the time series $b_{\ell}(t)$ of the expansion coefficients for each value of spherical harmonic degree $\ell$ :

$$
b_{\ell}(t)=\frac{1}{2}(2 \ell+1) \int_{-1}^{+1} \bar{B}(x, t) P_{\ell}(x) \mathrm{d} x .
$$

Since the power spectra of $b_{\ell}(t)$ show the power to be concentrated around the 22 yr resonance for the odd modes (cf. Stenflo and Vogel 1986; Stenflo and Guedel 1988), it is a good approximation to use a sinusoidal representation of the odd modes as

$$
b_{\ell}(t)=u_{\ell}+a_{\ell} \cos \left[\omega\left(t-t_{\ell}\right)\right],
$$

where $\omega=2 \pi / 22 \mathrm{yr}^{-1}$ and $-\omega t_{\ell}$ is the phase angle. $u_{\ell}$ represents a time-invariant, fossil field, while $a_{\ell}$ is the amplitude (constrained to be positive) of the mode with spherical harmonic degree $\ell$.

Using a 33 yr long data base (1959.6-1992.8) of Mt Wilson and Kitt Peak synoptic maps, the sinusoidal model of Eq. (27) has been applied with an iterative least squares technique to determine the three free parameters $u_{\ell}, a_{\ell}$, and $t_{\ell}$ for each $\ell$ (Stenflo 1994a).

The result for the amplitudes $a_{\ell}$ is shown in Fig. 17. It has a pronounced maximum at $\ell=5$. This harmonic degree dominates the behavior of the axisymmetric modes, not the dipole component. The "fossil" field component $u_{\ell}$ does not differ significantly from zero. The upper limit (1- $\sigma$ error) of the fossil dipole component $u_{1}$ is approximately $0.2 \mathrm{G}$. 
Fig. 18 Superposition of the sunspot butterfly diagram (thick solid contours) on the time-latitude distribution of the axisymmetric vertical magnetic field (thin contours), based on the spherical harmonic decomposition with the amplitudes given by the filled circles in Fig. 17 together with their corresponding phases

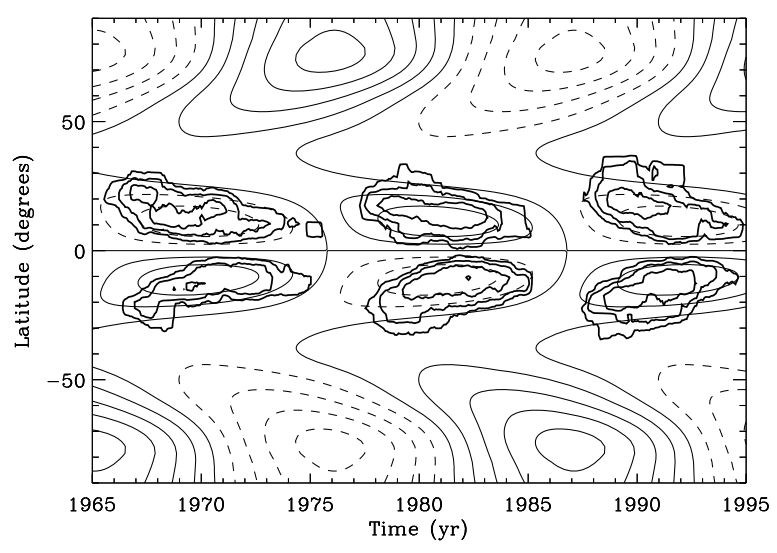

Using the amplitudes and phases for the seven odd modes (with $\ell=1-13$ ) determined with the model of Eq. (27), we can reconstruct the butterfly diagram. The result is shown in Fig. 18, where we for comparison have overplotted the isocontours for the sunspot number. The diagram is consistent with the full butterfly diagram of Fig. 16, although it does not show details on the smaller time scales, since it has been constructed exclusively from the $22 \mathrm{yr}$ contributions. In particular we do not see the herringbone pattern of discrete flux pulses that steeply migrate from the activity belts towards the poles, since they vary on a quasi-periodic biennial time scale.

\section{Magnetic intermittency}

When we zoom in on ever smaller scales on the quiet Sun, a fractal-like pattern with a high degree of self-similarity is revealed, as illustrated in Fig. 19. As we saw from the magnetic energy spectrum in Fig. 15, the magnetic structuring is expected to continue down to scales that are about four orders of magnitude smaller than the currently resolved scales.

Since all observations of quiet-sun magnetic fields represent averages over spatially unresolved structures, we make a distinction in our terminology between field strength and flux density, although both are measured in the same units. In the limit of infinite spatial resolution the two concepts are identical. Each resolution element averages over unresolved regions with different field strengths. The average field strength is the integrated flux divided by the area over which we integrate. We call this "flux density". Since the circular polarization is almost linearly related to the line-ofsight component of the field strength, the observed quantity in Stokes $V$ recordings is the line-of-sight component of the flux density. This is not the case for the linear polarization, since its relation to field strength is highly nonlinear (nearly quadratic). Therefore the quantity that is measured is related to the average energy density and not to flux density.

\subsection{Network and internetwork}

The frozen-in magnetic-field lines are carried by the convective motions to the cell boundaries. The pattern of flux concentrations therefore develops a cell structure. On 

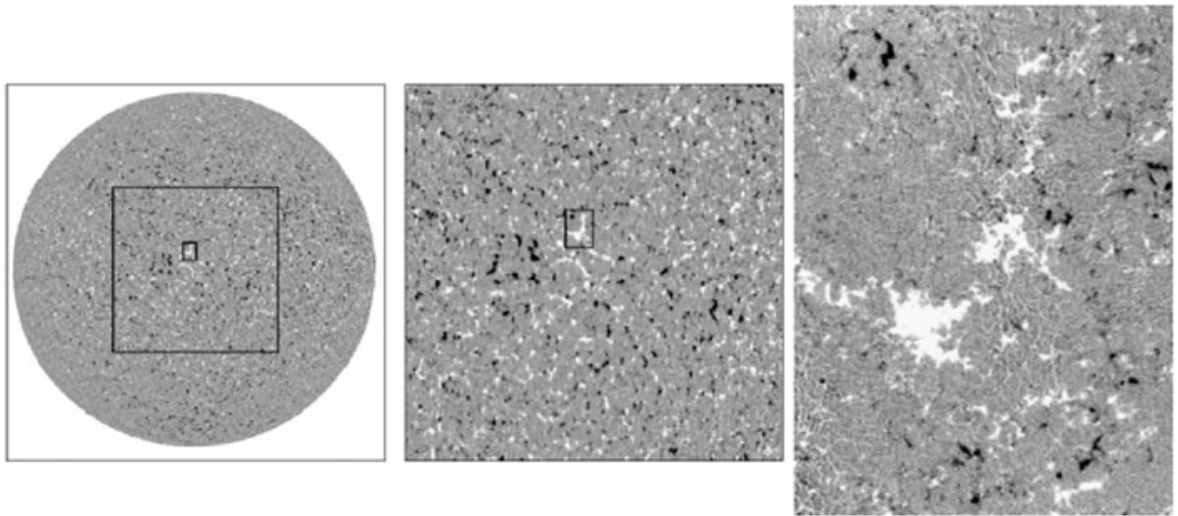

Fig. 19 Illustration of the fractal-like pattern of quiet-sun magnetic fields, recorded on February 9, 1996, when there were no spots present on the Sun's disk. The left panel shows the full-disk Kitt Peak magnetogram, the middle panel the magnified central portion of it. The right panel is a high-resolution recording at the Swedish La Palma Observatory (courtesy Göran Scharmer) of the small rectangular area marked near the middle of the other panels. The pattern is characterized by flux concentrations separated by voids with little net flux. Indirect evidence from Hanle depolarization measurements reveals that they are not voids at all but are seething with an "ocean" of turbulent fields with polarities mixed on scales beyond the resolution limit. From Stenflo (2010b)

the solar surface the two main types of velocity cells are represented by the granulation (cell size $\sim 1 \mathrm{Mm}$ ) and the supergranulation (cell size $\sim 30 \mathrm{Mm}$ ). The flux concentrations at the supergranular cell boundaries are conspicuous in magnetograms in the surroundings of active regions, where there is an abundance of magnetic flux, as illustrated in Fig. 20. In quiet regions only fragments of the cell boundaries are filled with flux, which makes the cell structure appear less obvious (cf. Figs. 14 and 19).

The term "network" that is often used for the flux concentrations at the supergranular cell boundaries was originally introduced to describe the brightness pattern in monochromatic images of the Sun recorded in various photospheric or chromospheric spectral lines. The brightness enhancements with implied heating of the upper atmosphere are, however, correlated both with the concentrations of magnetic flux and with downdrafts, since the largely horizontal flows in the interior of the supergranulation cells become vertical at the cell boundaries.

By definition, what is not network is called internetwork, supposed to represent the interior of supergranular cells. It should, however, be stressed that "network" and "internetwork" are statistical concepts, since supergranulation cells undergo statistical fluctuations, they form and dissolve over time scales of typically $24 \mathrm{hr}$. There is no way to tell whether a given magnetic element is part of the network or the internetwork. For this reason questions like whether large flux concentrations can also be found in the internetwork are not meaningful and cannot be answered. We always have a continuous distribution of flux densities of all magnitudes, there is no welldefined dichotomy between network and internetwork magnetic flux.

The absence of such a dichotomy in the observed flux densities becomes clear if we have a look at the histograms or probability density functions (PDF) of the 
Fig. 20 Recording with Hinode/SOT on September 27, 2012. The top panel is a G-band filtergram of active region $\mathrm{AR}$ 11575 , the bottom panel a line-of-sight magnetogram recorded with the NFI (Narrow-band Filter Imager) on SOT. Hinode is a Japanese mission developed and launched by ISAS/JAXA, with NAOJ as domestic partner and NASA and STFC (UK) as international partners. It is operated by these agencies in co-operation with ESA and NSC (Norway)
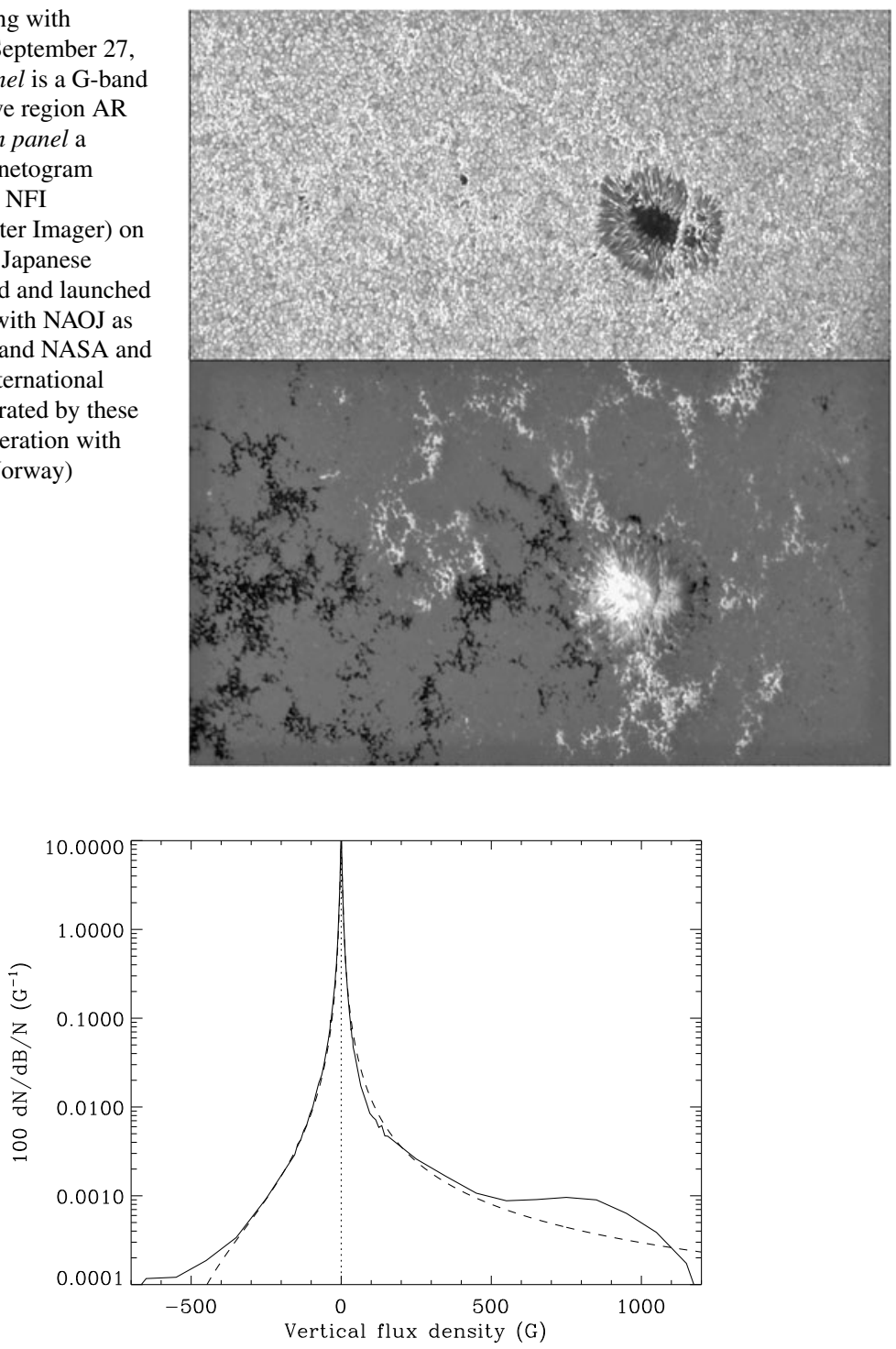

Fig. 21 Probability density function (PDF) for the vertical magnetic flux density at the disk center of the quiet Sun, as recorded by the SOT/SP instrument on Hinode on February 27, 2007 (solid line), from Stenflo (2010a). The Gaussian noise broadening has been removed. The dashed curve is an analytical representation with a core in the form of a stretched exponential and quadratically declining, extended wings. This PDF shape is typical for flux densities at resolved spatial scales. In the limit of infinite resolution we expect the field-strength PDF to have a 100-200 G wide core region in order to be consistent with the observational constraints from the Hanle effect (Stenflo 2012b)

measured flux densities, like in Fig. 21 derived from Hinode SOT/SP observations of the quiet-sun disk center. All such PDFs, after being corrected for noise broadening, are characterized by an extremely narrow and peaked core centered at zero flux den- 
sity, surrounded by slowly declining wings, which in observations with high spatial resolution (like with Hinode) may extend to $\mathrm{kG}$ flux densities (implying that such fields are nearly resolved). Since the quiet Sun exhibits large-scale patterns that are dominated by one of the two polarities, the PDF extracted for a given quiet region is generally asymmetric between plus and minus, like the one in Fig. 21. The bump that we see in the solid curve for large positive flux densities is not a statistically significant general property of the PDF shapes.

The dashed curve in Fig. 21 is an analytical representation that is characterized by a core region in the form of a stretched exponential and quadratically declining "damping" wings. It is typical for the shape of quiet-sun PDFs at resolved scales. The apparent scale invariance of this PDF shape must, however, end at very small scales (below about $10 \mathrm{~km}$ ) to be compatible with the observed Hanle depolarization (cf. Sects. 4.3 and 9.2), which reveals the existence of a hidden ocean of turbulent fields. It can be shown that the PDF damping wings cannot contribute significantly to the observed depolarization, which must instead originate in the PDF core region. A sufficiently large effect can only be obtained with a wide core region that has a half width of order 100-200 G (Stenflo 2012b). The PDF for the field strengths is therefore expected to be very different from the scale-dependent PDF for the flux densities.

The visual appearance of the magnetic pattern in Hinode magnetograms like the one in Fig. 20, which gives the impression that all the flux is in the network, with large empty voids in between, is simply a consequence of the choice of gray-scale cuts in the representation. These cuts are always set high enough to suppress the influence of the noisy PDF core region. All that is made visible then comes from the PDF damping wings, beyond typically $50 \mathrm{G}$. These parts of the PDF represent a tiny fraction of all the pixels in the image. The damping wings can be understood as signatures of intermittency, because the combination of high flux density and low occurrence probability implies spatial separation. The PDF core region on the other hand represents the apparent voids in the magnetograms.

\subsection{Extreme intermittency: kG-type flux on the quiet Sun}

The measured flux densities only represent lower limits to the actual field strengths. Assume for instance that instead of being resolved, the flux element that is the source of the measured polarization occupies only $1 \%$ of the resolution element. Then the actual or intrinsic field strength is 100 times larger than the apparent, average field strength or flux density.

To allow us to distinguish between these two scenarios we need another type of observable, which is provided by the magnetic line ratio. It is the ratio between the Stokes $V$ signals measured simultaneously in the line wings of the pair of Fe I lines at 5250.22 and $5247.06 \AA$. This particular line pair is the only known one for which the magnetic-field effects decouple from the thermodynamic effects so that intrinsic field strengths can be measured in a nearly model-independent way, independent of any particular assumption for the thermal structure of the atmosphere inside the flux concentrations. When the line-ratio technique was introduced four decades ago, it immediately led to the discovery that more than $90 \%$ of the magnetic flux visible 
Fig. 22 Sample spectrum of the 5247-5250 A region from the FTS Stokes $V$ atlas (Stenflo et al. 1984) of a weak plage at disk center

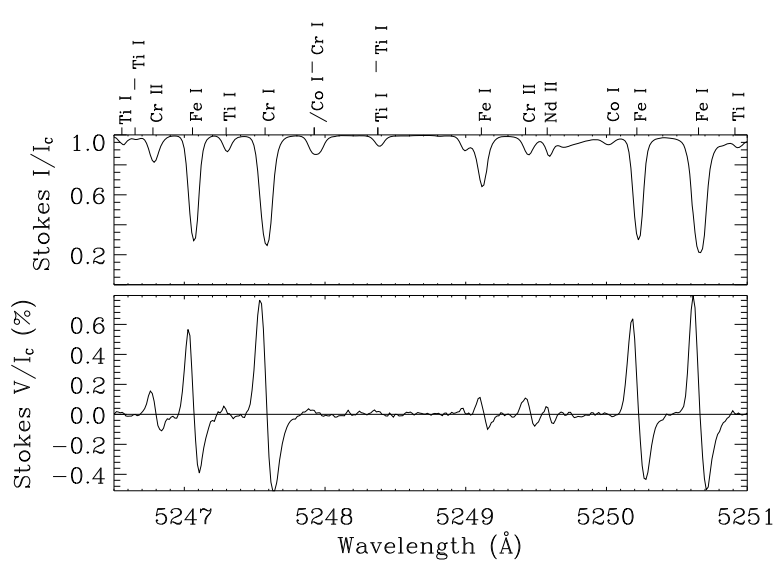

in magnetograms with moderate (a few arcsec) resolution has its origin in strong kG-type flux bundles (Stenflo 1973), usually referred to as flux tubes, which occupy a small fraction (typically $1 \%$ of the photospheric volume) (cf. also Howard and Stenflo 1972; Frazier and Stenflo 1972).

The idea behind the line-ratio technique is to make use of the deviation from linearity in the relation between Stokes $V$ and field strength when the Zeeman splitting becomes comparable to the line width. The differential nonlinearity (difference in amount of Zeeman saturation) between two lines that are identical in all respects except for their Landé factors is a direct function of intrinsic field strength. This differential effect expresses itself in the ratio between Stokes $V$ of the two lines. Since the magnetic filling factor divides out in the ratio, the effect is independent of the amount of measured flux and only depends on intrinsic field strength. $V$ alone gives the flux, so in combination with the $V$ ratio we get the filling factor.

Figure 22 shows the spectrally fully resolved $I$ and $V$ profiles for a small section of the FTS atlas of a weak plage at disk center that includes the 5250-5247 $\AA$ line pair, from the data set used in Stenflo et al. (1984). While the 5250.22 and $5247.06 \AA$ lines both belong to iron multiplet no. 1, have the same line strength and excitation potential, and are therefore formed in the same way in whatever atmosphere that we may have, they differ in their Landé factors, which are 3.0 and 2.0, respectively, both among the largest in the visible spectrum but significantly different from each other.

To illustrate how the differential nonlinearities affect the $V$ profiles, we superpose in Fig. 23 the $I$ and $V$ profiles of the 5250.22 $\AA$ line (solid curves) on top of the $5247.06 \AA$ line (dashed curves), whose $V$ profile has been scaled with the factor $3 / 2$ to compensate for the difference in Landé factor between the two lines. A common wavelength scale relative to the respective line centers has been used. Since for intrinsically weak fields $V \sim \partial I / \partial \lambda$ according to Eq. (11), we plot for comparison as the dotted curve $-\partial I / \partial \lambda$, normalized to the blue-lobe $V$ amplitude of the $5247.06 \AA$ line.

The upper panel verifies that the two Stokes $I$ profiles are indeed identical. The differential Zeeman saturation expresses itself in two ways. The $V$ amplitude of the line with the larger Landé factor gets suppressed (saturated) with respect to the other line, and the profile gets broadened and more extended. We notice that the blue lobe 


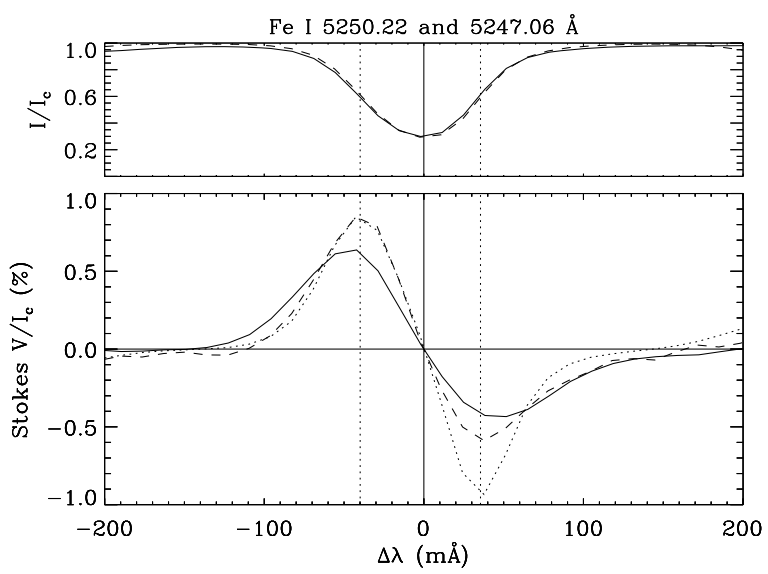

Fig. 23 Superposition of the $I$ and $V$ line profiles in Fig. 22 of the $5250.22 \AA$ line (solid) and $5247.06 \AA$ line (dashed) on a common wavelength scale relative to the respective line center. The Stokes $V$ profile of the $5247 \AA$ line has been scaled up by a factor of $3 / 2$ to compensate for the Landé factor difference with respect to the $5250.22 \AA$ line. The dotted curve represents $-\partial I / \partial \lambda$ for the $5247 \AA$ line, normalized to the blue-lobe Stokes $V$ amplitude. The locations of the two inflection points of the $I$ profile are indicated by the vertical dotted lines

of the $5247.06 \AA$ line has a shape that is nearly identical to that of $-\partial I / \partial \lambda$, indicating negligible Zeeman saturation for this line. The red $V$ lobes are both suppressed, in the same proportions, relative to the $-\partial I / \partial \lambda$ profile. While the areas of the blue and red $\partial I / \partial \lambda$ lobes are always exactly balanced (since integration must lead to the same continuum intensity on both sides of the line), they are significantly unbalanced for the $V$ profiles. Such a Stokes $V$ area asymmetry has its origin in correlations between the gradients along the line of sight of the magnetic field and the Doppler shifts (Illing et al. 1975; Auer and Heasley 1978; Stenflo et al. 1984; Steiner 2000).

We can now form the ratio between the Stokes $V 5250$ and $5247 \AA$ profiles in Fig. 23. This ratio profile is plotted in Fig. 24 vs. distance $|\Delta \lambda|$ from line center, separately for the blue lobe (solid curve) and the red lobe (dashed curve). We see that the blue and red lobe ratio profiles are in perfect agreement all the way from line center to far beyond the inflection points of the $I$ profile. They diverge only in the distant wings, where $V$ gets small and the red lobe is severely affected by the line-of-sight correlations between the magnetic- and velocity-field gradients.

If the fields were intrinsically weak ( $\lesssim 500 \mathrm{G})$, the $V$ ratio profile would not differ significantly from the horizontal level of unity. The more it differs from unity, the larger is the intrinsic field strength. The conversion of the line ratio to field strength is insensitive to the model atmosphere, as long as the chosen model (e.g. a simple Milne-Eddington atmosphere) is able to reproduce the half width of the intensity profile (since the magnitude of the line-ratio effect depends directly on the ratio between intrinsic Zeeman splitting and line width). Spatially unresolved line weakenings caused by a different thermodynamic structure of the magnetic elements have no effect as long as the line width does not change appreciably.

The observed line ratio reveals field strengths of order $1 \mathrm{kG}$ almost everywhere on the quiet Sun as observed with moderate spatial resolution. In contrast the measured 
Fig. 24 Ratio between the solid and dashed curves in Fig. 23 as a function of absolute wavelength distance from the line center. Solid line: blue lobe. Dashed line: red lobe. Dash-dotted line: theoretical line-ratio profile, based on a field-strength PDF from Stenflo (2012b) and an angular distribution from Stenflo (2010a), as described in the text. The vertical lines mark the locations of the Stokes $I$ inflection points for the blue (solid) and red (dashed) line wings

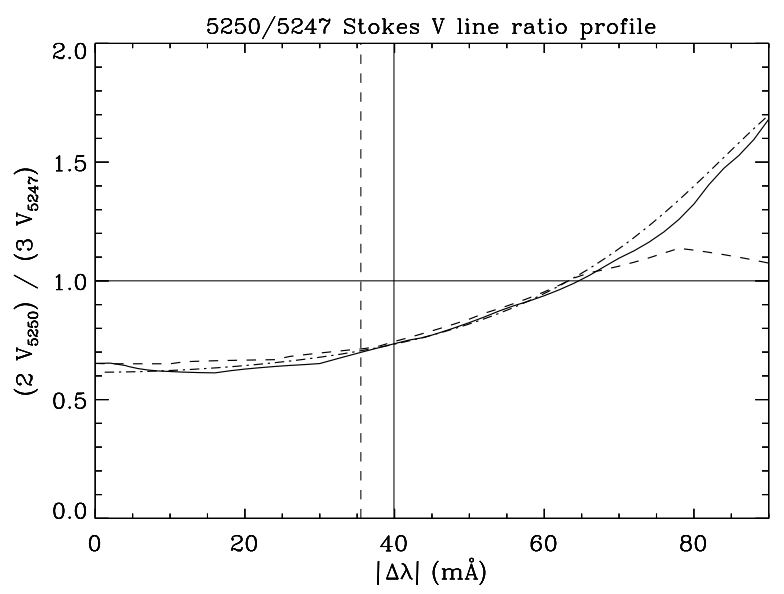

$V$ amplitudes correspond to flux densities (average field strengths) that are usually only a few G. These two findings can only be reconciled if the flux is extremely intermittent, with a mixture of very strong $(\mathrm{kG})$ and weak fields within the resolution element. The simplest description of this situation is in terms of a 2-component model, introduced as an interpretational tool in the initial applications of the lineratio method (Stenflo 1971, 1973). One component is magnetic with field strength $B$ and occupies a fraction $f$ (the filling factor) of the line-forming volume of the atmosphere (the angular resolution element times the depth of line formation along the line of sight). We stress here that the filling-factor concept relates to the volume and not to the surface area, because later applications of filling factors to horizontal fields and the transverse Zeeman effect have often been confused and incorrectly interpreted, due to a lack of understanding of the meaning of filling factors when the fields are highly inclined with respect to the solar surface. All confusion is eliminated if one understands that a filling factor is a volume occupation fraction.

In the standard application of the 2-component model the second component, with filling factor $1-f$, is assumed to be "nonmagnetic", because its contribution to the measured polarimetric signal is disregarded. It is obvious that this is an assumption made exclusively for mathematical and conceptual convenience. The extremely high electrical conductivity of the solar plasma effectively prohibits the existence of strictly nonmagnetic regions, any seed field will be tangled up and amplified by the turbulent motions. For instance, we now know from Hanle depolarization measurements that the "nonmagnetic" component is seething with an ocean of hidden, turbulent magnetic flux that may even dominate the energy balance of the solar atmosphere (Stenflo 1982; Trujillo Bueno et al. 2004). The 2-component model has, however, served as a very useful conceptual tool for the construction of a series of empirical flux tube models at increasing levels of sophistication (Solanki 1993). Typically these models consist of a magnetic element with axial symmetry that expands with height according to the laws of magnetohydrostatics, surrounded by nonmagnetic surroundings with inflows to a downdraft region at the outside of the flux tube.

Although the interpretation of the observed line-ratio profile in Fig. 24 in terms of a single-valued field strength (as implied by the 2-component model) is able to 
give a satisfactory fit to the observations, a much more realistic representation of the Sun is in terms of continuous distribution functions. For the derivation of the theoretical dash-dot fit curve in Fig. 24 we have therefore used distribution functions for both the field strengths (from Stenflo 2012b) and inclination angles according to their empirically determined statistical dependence on field strength (from Stenflo 2010a). Note that for the field-strength PDF one cannot use the distribution obtained for the flux densities (like the one in Fig. 21), but one needs a PDF that represents the case of infinite spatial resolution. The field-strength PDF inferred in Stenflo (2012b) has a broad core region needed to satisfy the Hanle constraint, and quadratically declining damping wings that extend out to $2 \mathrm{kG}$, where it is assumed that the PDF ends (because stronger fields cannot be in pressure balance with the ambient gas pressure). The calculations show that the PDF core region is nearly irrelevant to the line ratio, although it dominates the occurrence probability. The line ratio requires the existence of strong fields and gets its main contributions from the extended wings beyond about $500 \mathrm{G}$, although the integrated filling factor for these strong fields is small. We note that the theoretical fit in the far line wings improves when we account for the full anomalous Zeeman splitting pattern of the $5247.06 \AA$ line rather than treating it as a normal Zeeman triplet with an effective Landé factor.

\subsection{The 6302/6301 line ratio and its renormalization}

Although it is only for the Fe I 5250.22-5247.06 $\AA$ line pair that the magnetic-field effects (differential Zeeman saturation) can be cleanly separated from the thermodynamic and line-formation effects, useful applications with other line combinations are not ruled out. The Hinode SOT/SP instrument, which in recent years has delivered Stokes vector data that are by far the best in terms of high and stable angular resolution, is limited by design to the use of the Fe I 6302.5-6301.5 A line pair. The $V$ ratio of this pair is severely contaminated by thermodynamics and line-formation effects, since the two lines have different line strengths and are therefore formed at different heights in the atmosphere.

This contamination seriously complicates the interpretation and conversion of the line-ratio values into intrinsic field strengths. Khomenko and Collados (2007) tested the 6302/6301 line ratio through numerical simulations and found it to systematically give (if interpreted in a straightforward way) much too strong and generally unphysical values for the field strengths, in contrast to the 5250/5247 ratio, which behaved as expected. However, in scatter plots of $V$ for the $6302.5 \AA$ line vs. $V$ for the $6301.5 \AA$ line based on Hinode quiet-sun disk center data one finds a very well-defined linear regression relation with a slope that is clearly much smaller than expected if the fields were intrinsically weak. The small slope is a tell-tale signature of intrinsically strong fields. The same scatter plot, however, also reveals a small population of points with a different slope, consistent with the weak-field slope that one expects from scatter plots of $\partial I / \partial \lambda$ for the two lines. Assuming that the secondary population represents truly weak, "uncollapsed" fields, it is possible to recalibrate the 6302/6301 line-ratio scale to enable a determination of the intrinsic field strengths for the main flux population (Stenflo 2010a).

Most field strengths determined this way are of order $1 \mathrm{kG}$ and consistent with previous determinations based on the 5250/5247 line ratio. An upper limit to the size 
Fig. 25 Ratio between the "red" 6302/6301 Stokes $V / I$ line ratio and the corresponding "green" 5250/5247 line ratio as a function of center-to-limb distance. The curve allows the "contaminated" red line ratio to be translated to the green line ratio. From Stenflo et al. (2013)

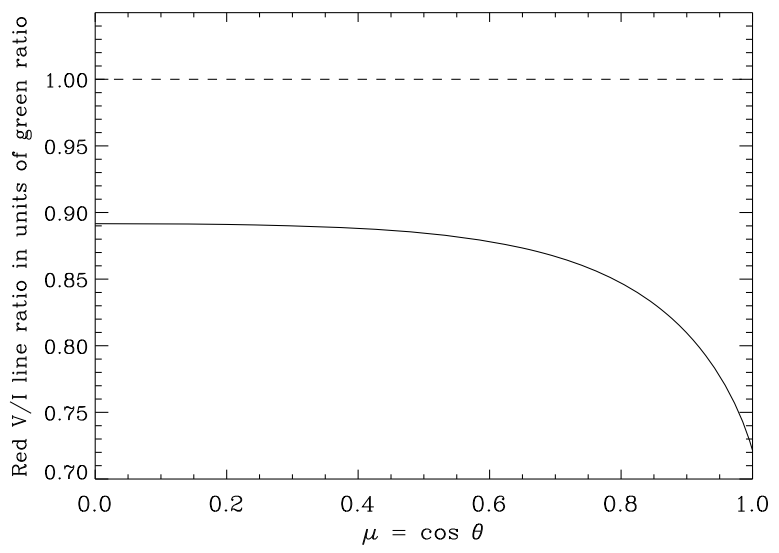

of the corresponding "collapsed" flux elements is obtained by assuming that there is one flux tube per resolution element. It is given by $\sqrt{f A}$, where $f$ is the filling factor and $A$ the area of the resolution element. If there is more than one flux tube per resolution element, then the size of each flux tube is naturally smaller. In this way it is possible to infer from the Hinode line-ratio data a histogram for the flux tube sizes. It shows that the main $\mathrm{kG}$-type flux tube population on the quiet Sun has sizes in the range 10-100 km (Stenflo 2011).

Recently it became possible to calibrate the contaminated 6302/6301 line ratio in terms of the uncontaminated 5250/5247 line ratio (Stenflo et al. 2013), using a special setup with the ZIMPOL polarimeter at IRSOL in Locarno, which allowed both line pairs to be recorded simultaneously on the same CCD sensor. The resulting "calibration curve" is shown in Fig. 25 as a function of center-to-limb distance. It means that the measured 6302/6301 line-ratio values have to be "renormalized" through division by the values represented by the solid line, before one can convert them into intrinsic field strengths, otherwise fictitiously high and unphysical field strengths would be found. At disk center the division factor is 0.72 , which agrees with the renormalization factor that was used in Stenflo (2010a) and based on the assumption that the secondary flux population in the scatter plot represented truly weak fields. The independent calibration represented by Fig. 25 provides a validation of the renormalization used.

\subsection{Convective collapse}

The $\mathrm{kG}$ fields that were revealed by the line-ratio technique four decades ago (Stenflo 1973) presented an enigma, since the dynamic forces of the convective flows that concentrate the flux at the cell boundaries are not strong enough to amplify the fields beyond the hectogauss range. The only force that is sufficiently strong to confine $\mathrm{kG}$ fields is the gas pressure. In a static situation the magnetic pressure is balanced by the difference in gas pressure between the exterior and interior of the magnetic flux element. If the flux region is entirely evacuated, the magnetic pressure equals the ambient gas pressure, which determines the maximum field strength that can be 
Fig. 26 The two main parameters that govern flux tube collapse, the photon mean free path and the superadiabatic temperature gradient, vs. height in the Sun's surface layers. The conditions favor a flux tube population in the $10-100 \mathrm{~km}$ size range, which is indicated by the horizontal dotted lines in the top panel. From Stenflo (2011)

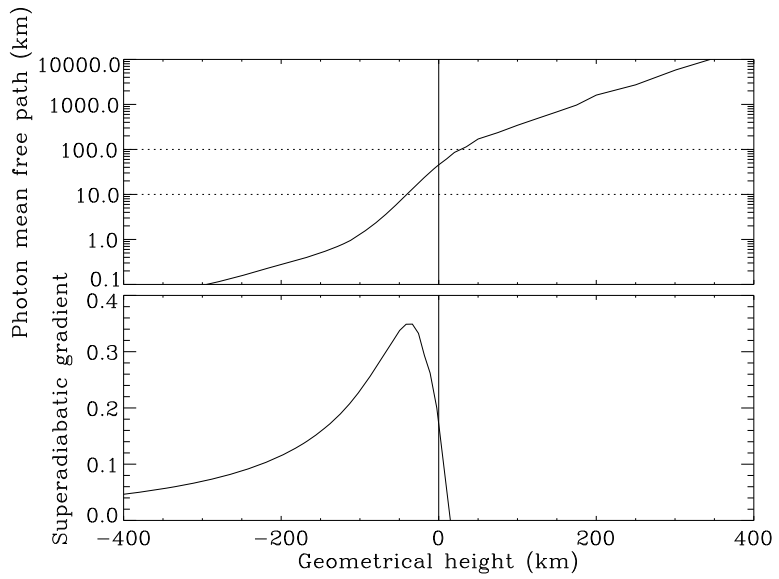

confined. For the photosphere it is of order $2 \mathrm{kG}$ but depends on the height level that we refer to.

The enigma was resolved by the mechanism of convective collapse, which was proposed in the late 1970s (Parker 1978; Spruit 1979; Spruit and Zweibel 1979; Unno and Ando 1979). It was developed and can best be described in terms of a 2-component scenario, with an initially weak magnetic flux element embedded in a nonmagnetic environment. It can be shown that this configuration is unstable in the superadiabatic region at the top of the convection zone (immediately below the photosphere). If the flux region is optically thick in the horizontal direction, an initially small downdraft will generate an adiabatic gradient inside the flux region, when the time scale for thermal exchange with the surroundings is larger than the convective time scale. This will create a temperature and pressure deficit with respect to the surroundings because of the external superadiabatic stratification. The deficit in gas pressure leads to compression, which amplifies the downdraft with the adiabatic cooling, causing evacuation of the flux region and spontaneous collapse. As a consequence the field gets concentrated until an equilibrium is reached and the external gas pressure is unable to amplify the field more.

The convective collapse instability is most efficient where the superadiabatic gradient is the largest, and the collapsing flux regions need to be optically thick, i.e., their cross sections need to be larger than the photon mean free path in the lateral direction across the flux tube. In Fig. 26 we show for an atmospheric model of Maltby et al. (1986) and a convection zone model of Spruit (1977) how the photon mean free path (in the continuum at $5000 \AA$ ) and superadiabatic gradient (difference between the magnitudes of the actual logarithmic temperature gradient and the corresponding adiabatic temperature gradient) vary with height. The zero point of the geometrical height scale, marked by the solid vertical line in the figure, is where the optical depth in the continuum at $5000 \AA$ is unity and represents the bottom of the photosphere. Below this level we enter the subsurface layers.

The superadiabatic gradient has a sharp maximum but drops to zero as we enter the photosphere, marking the end of the convection zone. In the near surface layers, where the convective collapse instability may occur, the photon mean free path is of 
order $10-100 \mathrm{~km}$, as indicated by the two dotted horizontal lines in the upper panel. Below this size range the instability will lose efficiency. We would therefore expect $\mathrm{kG}$ flux tubes to be larger than $d \approx 10 \mathrm{~km}$. After formation, flux tubes will have a limited life time proportional to $d^{2}$ due to the fluting instability (cf. Sect. 5.3) and will fragment to smaller scales, but kG-type fields may not be maintained below about $10 \mathrm{~km}$. Such theoretical considerations are consistent with the finding from line-ratio analysis of Hinode data that the bulk of the collapsed flux population has sizes in the range 10-100 km (Stenflo 2011).

Although most of the collapsed flux tubes are expected to have sizes just beyond the resolution capabilities of current telescopes, the distribution has a tail that extends well into the resolved domain. Thus it has been possible to directly resolve such flux tubes on the quiet Sun (cf. Keller 1992; Lagg et al. 2010). The convective collapse process has received support both from observations (Nagata et al. 2008; Fischer et al. 2009) and from 3-D MHD simulations (Danilovic et al. 2010).

It should be noted that because the theoretical scenario that describes the convective collapse mechanism uses the idealization of a magnetic flux region embedded in nonmagnetic surroundings, the spontaneous instability leads to a dichotomy, where the flux ends up being in either collapsed or uncollapsed form. This scenario has its counterpart in the 2-component interpretational model used for the line-ratio observations. In the real Sun, however, there is no sharp distinction between magnetic and nonmagnetic, the conditions are better described in terms of continuous distribution functions connecting the weak and strong fields. In such an environment the occurrence of the collapse instability is not an either-or question, and the amplitude and end result of the instability can be expected to have a continuous range of values.

\section{Small-scale properties of internetwork magnetic fields}

The magnetic intermittency described in the previous section implies that much of the photospheric magnetic flux exists in highly concentrated form. As these flux elements have small filling factors, the bulk of the photosphere must be filled with much weaker fields, which get tangled by the turbulent motions and as a consequence become nearly invisible in magnetograms because of cancelation effects, unless the angular resolution is sufficiently high to separate the opposite magnetic polarities. In the present section we will focus the attention on the properties of these weaker, tangled fields, which with commonly used terminology may be classified as "internetwork" fields, in contrast to the collapsed network-type fields that were dealt with in the previous section.

We will first address the angular distribution of the internetwork fields and then deal with their intrinsic field strengths. Since most of the structuring of these fields takes place on scales far smaller than the resolved ones, the diagnostic methods used need to be resolution independent and be based on statistical ensemble averages. The determination of the angular distributions is based on the symmetry properties of the transverse Zeeman effect, the determination of the field strengths on observations of the Hanle depolarization effect. 


\subsection{Angular distribution of the field vectors}

In an isotropic medium the angular distribution of the field vectors would also be isotropic. The stratification of the outer solar envelope with its nearly exponential decrease of the gas pressure with height has, however, a number of important consequences for the field orientation: (a) The flux tubes that are anchored in the deeper and denser layers are being pushed towards a vertical orientation by buoyancy. (b) The exponential decrease of the external gas pressure leads to an expansion with height of the flux tubes, whose field lines flare out to become increasingly horizontal with a canopy structure in the higher layers (Giovanelli 1980; Jones and Giovanelli 1983). (c) The many flux loops of various sizes, which represent the topological structure of the turbulent field, are predominantly vertical in the deeper layers where their footpoints are anchored, but are more horizontal near the loop tops (cf. Steiner 2010). For these various reasons we expect the angular distribution of the field vectors on the quiet Sun to have a preference for the vertical orientation in the lower layers, but the preference should shift in favor of the horizontal orientation as we move up in height. However, we need observations to verify this expectation and to tell us at what height the transition from vertical to horizontal preference takes place and how it depends on field strength.

It came as a big surprise when initial analysis of Hinode SOT/SP data for the quietsun disk center was proclaimed to show that the horizontal fields not only dominate, but that there is as much as five times more horizontal than vertical magnetic flux (Orozco Suárez et al. 2007; Lites et al. 2008). Subsequent analyses of the identical Hinode data set, however, gave completely different results, quasi-isotropic angular distribution according to Asensio Ramos (2009), vertical preference but with a fluxdensity dependence that approaches the isotropic case in the weak flux-density limit according to Stenflo (2010a).

The cause of this colossal divergence of the results has to do with the pitfalls when trying to determine the field inclinations from a combination of the linear- and circular-polarization data. In contrast to the circular polarization, the linear polarization has a highly nonlinear (nearly quadratic) dependence on the magnetic field (cf. Sect. 3). The interpretation of the optical averages over each pixel depends on the model that we use for the subpixel structuring of the nonlinear quantity over which we average. In addition the polarimetric noise is larger in the transverse field in comparison with the longitudinal field by a factor of approximately 25 (cf. Fig. 5). The results for the inclination angles then depend on how we deal with both the noise and the nonlinearities.

Recently the angular distribution could be determined with a method that avoids these pitfalls by not depending on any comparison between the linear and circular polarization but only on the qualitative symmetry properties of the Stokes line profiles in linear polarization due to the transverse Zeeman effect when observing off disk center. To understand these symmetry properties, let us begin by considering a vertical magnetic field that is observed relatively close to the solar limb, and choose the Stokes coordinate system such that the positive $Q$ direction is defined to be perpendicular to the radius vector on the solar disk (or, equivalently, parallel to the nearest solar limb). As in the vertical field case the transverse component is along the radius 
Fig. 27 Signature of nearly vertical fields for a polar facula at $\mu=0.108$ ( 6 arcsec inside the limb) near the heliographic $S$ pole. As the Stokes coordinate system has been defined such that positive Stokes $Q$ represents linear polarization oriented perpendicular to the radius vector, the symmetric 3-lobe pattern of the transverse Zeeman effect in Stokes $Q$ will have the sign pattern -+- if the field is vertical. The recording was made with ZIMPOL-2 at the French THEMIS telescope on June 10, 2008. From Stenflo (2013)
Small facula near $\mathrm{S}$ pole, $\mu=0.108$
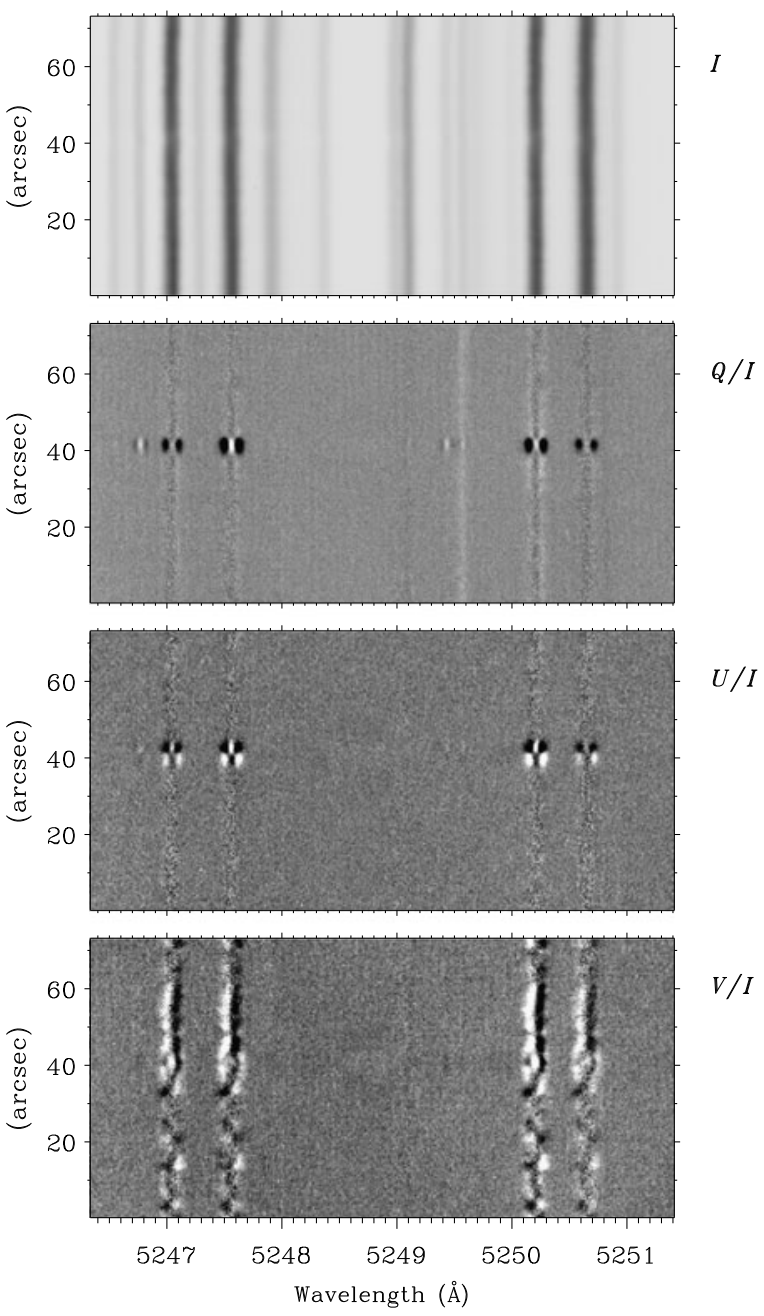

vector, the $Q$ polarization of the central $\pi$ component of the line profile will be positive for an absorption line. The observed, symmetric Stokes $Q$ line profile will then have the sign pattern - + - This is illustrated in Fig. 27 for a small polar facula 6 arcsec inside the S polar limb. All spectral lines in the field of view exhibit the $Q / I$ sign pattern -+- , a tell-tale signature of vertical fields. $U / I$, which relates to the $\pm 45^{\circ}$ directions of the transverse field, is nonzero, indicating that the facular field is not strictly vertical but has an angular distribution, but $U / I$ reverses sign across the facula with a balance between the opposite signs.

The weak, emission-like feature in $Q / I$ at $5249.58 \AA$ is due to nonmagnetic scattering polarization in ionized neodymium and is unrelated to the present topic.

For transverse fields perpendicular to the radius vector, which would represent horizontal fields, the $Q / I$ sign pattern would be the opposite, +-+ . We notice in 
Fig. 27 that this sign pattern, although faint, characterizes everything along the slit outside the polar facula.

Let us now instead of fields with a well-defined orientation consider a statistical ensemble of magnetic elements with an angular distribution of the field vectors that is axisymmetric around the vertical direction. Then the distribution of inclination angles determines whether the overall distribution, in comparison with the isotropic case, is preferentially vertical (more peaked around the vertical) or preferentially horizontal (more pancake like). It can be shown (cf. Stenflo 2013) that whenever there is any vertical preference, even if it is slight, then the $Q / I$ sign pattern of the ensemble average will always be -+- , while a horizontal preference will produce the pattern +-+ for the ensemble average. Therefore we can conclude that the weak background fields (which may be classified as internetwork fields) in Fig. 27 are preferentially horizontal.

We have made a number of recordings across different types of flux concentrations at various center-to-limb distances, from tiny faculae to small sunspots, and all of them exhibit the $Q / I-+-$ sign pattern, which tells us that they have a clear vertical preference for all disk positions, all the way to the limb. The behavior of the weak background fluxes is different, as we saw in Fig. 27. We have therefore also made recordings (with the Swiss ZIMPOL-2 polarimeter mounted on the French THEMIS telescope on Tenerife) in a sequence of very quiet regions spanning a range of limb distances from $\mu=0.08$ to $\mu=0.5$. None of these regions included any facular or network element. All of them can therefore be classified as internetwork. The ensemble averages needed to determine whether we have vertical or horizontal preference are obtained by averaging over the spatial resolution elements along the spectrograph slit. The recordings reveal a systematic variation of the $Q / I$ sign pattern with $\mu$, with a transition from one sign pattern to the opposite at $\mu \approx 0.2$. Closer to the limb there is a horizontal preference, closer to disk center a vertical preference.

Since smaller $\mu$ values refer to higher levels of the solar atmosphere, we can therefore conclude that the internetwork field favors a vertical orientation in the low to middle photosphere but has a transition in favor of the horizontal orientation above the atmospheric level that corresponds to $\mu=0.2$. In contrast, the network and facular fields preserve a vertical preference over the entire height range spanned by the various $\mu$ values.

Note that this result is model independent in the sense that it only depends on the qualitative symmetry property of the transverse Zeeman effect represented by the sign pattern of the $Q / I$ profiles. It is also resolution independent, since it only refers to ensemble averages, and optical and numerical averagings are equivalent. If we average the $Q$ signal for a given area of the Sun optically because it is not resolved (and thus represents one resolution element), or we resolve the same area with many resolution elements and then do numerical averaging of $Q$ for each of them, the results will be identical. $Q$ is proportional to the number of photons from each surface element, regardless of whether the element is resolved or not. Therefore our result for the angular distribution will not change with the application of future telescopes that have much higher resolving power.

The idea of using ensemble averages of Stokes $Q$ to determine the angular distributions for observations off disk center was introduced long ago (Stenflo 1987), 
but its application was limited at that time, since the observations used single-pixel detectors (photomultipliers). With the present availability of high-precision imaging polarimeters we are now in a position to exploit this technique in great detail.

Close to the solar limb the properties of the horizontal magnetic fields in the upper photosphere can also be explored with circular-polarization magnetograms, since near the limb the line of sight is nearly horizontal. Thus the circular polarization as observed with low spatial resolution ( $3 \mathrm{arcsec})$ is found to exhibit large temporal fluctuations, which reveal that the horizontal components of the internetwork magnetic fields are highly dynamic (Harvey et al. 2007).

\subsection{Intrinsic field strengths of the internetwork fields}

In Sect. 4.3 we described how observations of the Hanle effect reveal the existence of an ocean of hidden magnetic flux with mixed polarities on scales much smaller than the spatial resolution of current telescopes. The scattering polarization in photospheric lines is observed to be systematically reduced and spatially almost invariant, while the orientation of the plane of polarization does not deviate significantly from the nonmagnetic case. The only known consistent explanation is in terms of the Hanle effect. The reduced degree of polarization requires an abundance of magnetic fields with Zeeman splittings comparable in magnitude to the combined collisional and radiative damping widths of the atomic transitions used for the diagnostics. The absence of any observed Hanle rotation of the plane of polarization that one would expect for such fields can only be explained by cancelation effects of positive and negative rotation angles when averaging over an ensemble of spatially unresolved flux elements (Stenflo 1982). Note that this behavior only applies to photospheric but not to chromospheric lines. The scenario with an ocean of hidden, tangled magnetic flux is only valid for the photospheric levels, not above.

The conversion of Hanle depolarization to turbulent field strength $B_{t}$ was indicated in Fig. 7. Usually the flux ensemble is for simplicity characterized by a single field strength, while the angular distribution is assumed to be isotropic, although the obtained results are not very sensitive to the assumed field orientations. The first applications of the Hanle depolarization effect allowed $B_{t}$ to be constrained within the range 10-100 $\mathrm{G}$ (Stenflo 1982, 1987). This range is still valid in spite of rather divergent results since then with detailed radiative-transfer modeling.

Most of the modeling has focused on the interpretation of observations with the Sr I 4607 A line. While initial radiative-transfer modeling gave values of order $30 \mathrm{G}$ (Faurobert-Scholl 1993; Faurobert-Scholl et al. 1995), the most sophisticated modeling to date with 3-D atmospheres generated by numerical simulations gave $60 \mathrm{G}$ (Trujillo Bueno et al. 2004). When the interpretation is made with plausible PDF field strength distributions instead of the single-valued assumption, the Hanle constraints imply an average field strength about twice as large, suggesting that the total magnetic energy density of the hidden field may play an important role for the overall energy balance of the atmosphere (Trujillo Bueno et al. 2004).

These large $B_{t}$ values are in apparent contradiction with interpretations based on observations of the differential Hanle effect in pairs of optically thin molecular lines, like the $C_{2}$ lines around $5141 \AA$ (Berdyugina and Fluri 2004). A synoptic program 
with this line pair that was started in 2007 with ZIMPOL at IRSOL (Istituto Ricerche Solari Locarno) has given the value $B_{t}=7.4 \pm 0.8 \mathrm{G}$ for the period 2007-2009, with no evidence for any significant temporal variations (Kleint et al. 2010).

Trujillo Bueno et al. (2004) have offered a possible explanation for this apparent contradiction, by demonstrating that the molecular $\mathrm{C}_{2}$ lines are formed exclusively inside the granulation cells, while the Sr I $4607 \AA$ Aine gets similar contributions from both the intergranular lanes and the cell interiors. This implies that the $B_{t}$ value inside the intergranular lanes must be substantially higher than the $60 \mathrm{G}$ value, since the cell interiors with their large filling factors would not contribute much to the observed Hanle depolarization.

This interpretation is plausible, since the intermittent, collapsed kG-type flux concentrations are found to have a strong preference for the intergranular lanes as well (Stenflo 2011), and there is reason to believe that the tangled fields are supplied with flux from the decaying flux tube fields. Observational support has been provided by Snik et al. (2010), who analysed Hinode SOT high-resolution observations with a CN filter and were able to statistical separate the cell interiors and the intergranular lanes. It was found that the scattering polarization was significantly reduced in the lanes. Detailed radiative-transfer modeling of the Hanle effect in the $\mathrm{CN}$ ultraviolet lines leads to values for $B_{t}$ that are comparable in magnitude to those found for the $\mathrm{Sr} I$ $4607 \AA$ line (Shapiro et al. 2011). The difference with respect to the optically thin $C_{2}$ lines is explained by the much larger optical thickness of the $\mathrm{CN}$ lines, which makes them vary less from cell interior to the lanes. In spite of these insights we need Hanle effect observations in various types of spectral lines with high spatial resolution to better clarify these issues.

Another not yet settled issue is whether the hidden flux revealed by the observed Hanle depolarization varies with the phase of the solar cycle. The synoptic observations made so far in the $\mathrm{C}_{2}$ lines suggest that the field is invariant. Trujillo Bueno et al. (2004) have compiled evidence, which indicates that also the depolarization in the Sr I $4607 \AA$ line does not vary with the cycle. However, visual inspection of recordings of various sections of the Second Solar Spectrum observed since 1995 indicates that the appearance of the spectrum is subject to significant changes, which imply that there are substantial cycle variations of the hidden field (Stenflo 2003). We therefore need a synoptic program not only for the $\mathrm{C}_{2}$ lines, but in particular also for atomic lines like the Sr I $4607 \AA$ line, with which such large strengths for the hidden field are obtained. Such a program is currently being initiated with ZIMPOL at IRSOL.

In Sect. 6 it was noted in Fig. 15 in the context of observational evidence for the operation of a local dynamo that a Kolmogorov-type $-5 / 3$ power law in the inertial range that goes down to the magnetic diffusion limit is much too steep to provide the large amounts of small-scale magnetic flux that are required in order to satisfy the constraints from the observed Hanle depolarization. Consistency with the observed Hanle effect can be restored if the small-scale end of the energy spectrum is raised to give a slope of -1.0 instead. Such a pile-up of small-scale flux cannot be naturally explained in terms of the turbulent cascade from larger-scale fluxes generated by the global dynamo but seems to demand the existence of an additional source of flux, which would be naturally provided by a local dynamo. 
If the hidden flux revealed by the Sr I $4607 \AA$ line observations is due to a local dynamo, then it must be statistically invariant with respect to the solar cycle. If on the other hand its source comes from the global dynamo, then significant cycle variations are unavoidable. Therefore a synoptic Hanle program for atomic lines should be able to settle this issue.

\section{Concluding remarks}

New powerful solar facilities, both on ground and in space, are being implemented or are in construction. A primary objective of most of these facilities is the diagnostics of magnetic fields via spectro-polarimetry with high spatial resolution, to advance our understanding of the small-scale morphology and evolution of the magnetic field and its relation to the thermodynamic structuring and solar activity. It is believed that many of the fundamental processes take place on small scales, and that it is therefore crucial to resolve these scales to understand the underlying physics. The enormous advances in computing power now allow numerical simulations of the stratified solar atmosphere with the inclusion of all the relevant radiative and MHD processes and a spatial resolution in 3-D that surpasses that of the observations. Progress is often judged by how well the simulations compare with the observations.

In the present review we have paid particular attention to the property that the magnetic structuring continues to scales that are orders of magnitude smaller than the resolution of current telescopes and also much smaller than the grid resolution of the numerical simulations. In addition, as the current telescope resolution is close to the transition around the $100 \mathrm{~km}$ scale between the optically thick and thin regimes, there is a more fundamental limitation to the resolution that can be reached by future telescopes. While there is no principal limitation to the possible angular resolution in the transversal plane, the resolution along the line of sight is fixed by the optical depth scale and remains of order $100 \mathrm{~km}$ in the photosphere, no matter what the angular resolution is. The structuring through tangling of the field lines or through local dynamo action will ignore this limit and continue far into the optically thin regime. The observational consequence of this is that the visibility of structures in magnetograms will decline with smaller scale size due to cancelation effects or small filling factors along the line of sight.

Here we have highlighted three types of techniques that avoid the cancelation and filling-factor problems and lead to resolution-independent fundamental insights into the nature and properties of the magnetic fields in the spatially unresolved small-scale domain: (1) Magnetic line-ratio technique to explore the magnetic intermittency and intrinsic field strengths in the strong-field tail of the probability density function, without dependence on filling factor. (2) Exclusive use of the symmetry properties of the transverse Zeeman effect in observations away from disk center, to determine whether the angular distribution of the field vectors favors the vertical or horizontal orientation in comparison with the isotropic case. (3) Use of the Hanle effect depolarization of photospheric spectral lines, to determine the intrinsic strength of a field that is tangled with mixed polarities on scales in the optically thin regime.

Instead of trying to invert the above polarimetric observables with the help of idealized interpretational models, more physical insight may be gained by going in 
the opposite direction, namely to test the validity of the MHD simulations of the solar atmosphere by computing synthetic values for the observables that represent the three mentioned techniques used to diagnose the resolution-independent properties. This has not yet been done. A particularly fundamental test is to try to reproduce the observed Hanle depolarization in lines like the Sr I $4607 \AA$ line. If as believed much of the Hanle depolarization effect occurs on scales beyond the reach of the grid resolutions of state-of-the-art simulations, then these simulations are likely to fail to reproduce this observable. It is a prediction that can be directly tested with available data and simulation models. Another fundamental test is to try to reproduce the observed sign pattern of the ensemble averages of the transverse Zeeman effect as a function of center-to-limb distance. Still another test is the reproduction of the observed 5250/5247 Stokes $V$ line ratio, in particular its small intrinsic scatter and its calibrated relation to the $6302 / 6301$ line ratio.

The present review has focused on the photosphere, since almost all the knowledge gained from Stokes polarimetry about the magnetic-field properties refer to this part of the atmosphere. Future work will increasingly focus on the chromosphere and the atmosphere above, where Zeeman-effect techniques are less effective. Here we expect the diagnostic tools provided by the Hanle effect via the wealth of scattering polarization structures in the Second Solar Spectrum to come increasingly into the foreground. The theoretical tools to deal with observations of scattering polarization in magnetized media are currently under development (cf. the references in Sect. 1 to the series of Solar Polarization Workshops). This work in progress is addressing and trying to solve deep problems related to the quantum-mechanical foundation of the interaction between radiation and matter in magnetized media with scattering and collisional processes. These processes need to be integrated in a polarized radiativetransfer formulation with partial frequency redistribution, which then needs to be applied to atmospheres with spatially unresolved magnetic structures. The Sun serves as a fascinating quantum and plasma physics laboratory for these various phenomena.

When high-resolution spectro-polarimetric observations from space of the chromosphere-corona transition region will become available in the future, the theoretical foundations developed in the context of the Second Solar Spectrum may be extended to the vacuum ultraviolet part of the spectrum and applied to the diagnostics of magnetic fields in the regions of the Sun's atmosphere, where the corona is being heated.

\section{References}

Altschuler MD, Trotter DE, Newkirk G Jr, Howard R (1974) The large-scale solar magnetic field. Sol Phys 39:3-17. doi:10.1007/BF00154967

Anusha LS, Nagendra KN, Bianda M, Stenflo JO, Holzreuter R, Sampoorna M, Frisch H, Ramelli R, Smitha HN (2011) Analysis of the Forward-scattering Hanle Effect in the Ca I $4227 \AA$ Line. Astrophys J 737:95. doi:10.1088/0004-637X/737/2/95

Asensio Ramos A (2009) Evidence for quasi-isotropic magnetic fields from Hinode quiet-Sun observations. Astrophys J 701:1032. doi:10.1088/0004-637X/701/2/1032

Auer LH, Heasley JN (1978) The origin of broad-band circular polarization in sunspots. Astron Astrophys 64:67-71

Babcock HW (1953) The solar magnetograph. Astrophys J 118:387

Babcock HW (1961) The topology of the Sun's magnetic field and the 22-year cycle. Astrophys J 133:572587. doi:10.1086/147060 
Baur TG, House LL, Hull HK (1980) A spectrum scanning Stokes polarimeter. Sol Phys 65:111-146. doi: $10.1007 / \mathrm{BF} 00151388$

Berdyugina SV, Fluri DM (2004) Evidence for the Hanle effect in molecular lines. Astron Astrophys 417:775-784. doi:10.1051/0004-6361:20034452

Berdyugina SV, Nagendra KN, Ramelli R (eds) (2009) In: Solar polarization 5: in honor of Jan Olof Stenflo. Astronomical society of the pacific conference series, vol 405

Bianda M, Ramelli R, Anusha LS, Stenflo JO, Nagendra KN, Holzreuter R, Sampoorna M, Frisch H, Smitha HN (2011) Observations of the forward scattering Hanle effect in the Ca I $4227 \AA$ line. Astron Astrophys 530:L13. doi:10.1051/0004-6361/201117047

Bommier V, Sahal-Brechot S (1978) Quantum theory of the Hanle effect-calculations of the Stokes parameters of the D3 helium line for quiescent prominences. Astron Astrophys 69:57-64

Brandenburg A (2005) The case for a distributed solar dynamo shaped by near-surface shear. Astrophys J 625:539. doi:10.1086/429584. arXiv:astro-ph/0502275

Brandenburg A, Subramanian K (2005) Astrophysical magnetic fields and nonlinear dynamo theory. Phys Rep 417:1-209. doi:10.1016/j.physrep.2005.06.005. arXiv:astro-ph/0405052

Buehler D, Lagg A, Solanki SK (2013) Quiet Sun magnetic fields observed by Hinode: support for a local dynamo. Astron Astrophys 555:A33. doi:10.1051/0004-6361/201321152

Casini R, Lites BW (eds) (2006) In: Solar polarization 4. Astronomical society of the pacific conference series, vol 358

Cattaneo F (1999) On the origin of magnetic fields in the quiet photosphere. Astrophys J Lett 515:L39L42. doi:10.1086/311962

Charbonneau P (2010) Dynamo models of the solar cycle. Living Rev Sol Phys 7:3. doi:10.12942/lrsp2010-3

Danilovic S, Schüssler M, Solanki SK (2010) Magnetic field intensification: comparison of 3D MHD simulations with Hinode/SP results. Astron Astrophys 509:A76. doi:10.1051/0004-6361/200912283

Dasi-Espuig M, Solanki SK, Krivova NA, Cameron R, Peñuela T (2010) Sunspot group tilt angles and the strength of the solar cycle. Astron Astrophys 518:A7. doi:10.1051/0004-6361/201014301

del Toro Iniesta JC (2003) Introduction to spectropolarimetry. Cambridge University Press, Cambridge

DeRosa ML, Brun AS, Hoeksema JT (2012) Solar magnetic field reversals and the role of dynamo families. Astrophys J 757:96. doi:10.1088/0004-637X/757/1/96

Elsasser WM (1946) Induction effects in terrestrial magnetism. Part I. Theory. Phys Rev 69:106-116

Elsasser WM (1956) Hydromagnetic dynamo theory. Rev Mod Phys 28:135-163

Faurobert-Scholl M (1993) Investigation of microturbulent magnetic fields in the solar photosphere by their Hanle effect in the Sr I 4607 A line. Astron Astrophys 268:765-774

Faurobert-Scholl M, Feautrier N, Machefert F, Petrovay K, Spielfiedel A (1995) Turbulent magnetic fields in the solar photosphere: diagnostics and interpretation. Astron Astrophys 298:289

Fischer CE, de Wijn AG, Centeno R, Lites BW, Keller CU (2009) Statistics of convective collapse events in the photosphere and chromosphere observed with the Hinode SOT. Astron Astrophys 504:583-588. doi:10.1051/0004-6361/200912445

Frazier EN, Stenflo JO (1972) On the small-scale structure of solar magnetic fields. Sol Phys 27:330-346. doi: $10.1007 / \mathrm{BF} 00153105$

Gandorfer A (2000) The second solar spectrum: a high spectral resolution polarimetric survey of scattering polarization at the solar limb in graphical representation. Volume I: $4625 \AA$ to $6995 \AA$ A vdf Hochschulverlag, Zurich

Gandorfer A (2002) The second solar spectrum: a high spectral resolution polarimetric survey of scattering polarization at the solar limb in graphical representation. Volume II: $3910 \AA$ to $4630 \AA$ Adf Hochschulverlag, Zurich

Gandorfer A (2005) The second solar spectrum: a high spectral resolution polarimetric survey of scattering polarization at the solar limb in graphical representation. Volume III: $3160 \AA$ to $3915 \AA$. vdf Hochschulverlag, Zurich

Gandorfer AM, Steiner P, Povel HP, Aebersold F, Egger U, Feller A, Gisler D, Hagenbuch S, Stenflo JO (2004) Solar polarimetry in the near UV with the Zurich Imaging Polarimeter ZIMPOL II. Astron Astrophys 422:703-708. doi:10.1051/0004-6361:20040254

Giovanelli RG (1980) An exploratory two-dimensional study of the coarse structure of network magnetic fields. Sol Phys 68:49-69. doi:10.1007/BF00153266

Hale GE (1908) On the probable existence of a magnetic field in sun-spots. Astrophys J 28:315. doi:10. $1086 / 141602$ 
Hale GE, Seares FH, van Maanen A, Ellerman F (1918) The general magnetic field of the Sun. Apparent variation of field-strength with level in the solar atmosphere. Astrophys J 47:206-254. doi:10. $1086 / 142403$

Hale GE, Ellerman F, Nicholson SB, Joy AH (1919) The magnetic polarity of sun-spots. Astrophys J 49:153-178. doi:10.1086/142452

Hanle W (1924) Über magnetische Beeinflussung der Polarisation der Resonanzfluoreszenz. Z Phys 30:93-105

Harvey J, Livingston W, Slaughter C (1972) A line-profile Stokesmeter: preliminary results on non-sunspot fields. In: Line formation in the presence of magnetic fields. HAO/NCAR, Boulder, pp 227-250

Harvey JW, Branston D, Henney CJ, Keller CU (SOLIS and GONG Teams) (2007) Seething horizontal magnetic fields in the quiet solar photosphere. Astrophys J Lett 659:L177-L180. doi:10.1086/518036. arXiv:astro-ph/0702415

Harvey KL (1993) Magnetic bipoles on the Sun. PhD thesis, Univ Utrecht

Harvey KL, Martin SF (1973) Ephemeral active regions. Sol Phys 32:389-402. doi:10.1007/BF00154951

Harvey KL, Zwaan C (1993) Properties and emergence of bipolar active regions. Sol Phys 148:85-118. doi:10.1007/BF00675537

Harvey KL, Harvey JW, Martin SF (1975) Ephemeral active regions in 1970 and 1973. Sol Phys 40:87102. doi:10.1007/BF00183154

Hasan SS, Rutten RJ (eds) (2010) Magnetic coupling between the interior and atmosphere of the Sun. doi:10.1007/978-3-642-02859-5

House LL (1970a) The resonance fluorescence of polarized radiation. I. The general scattering function. J Quant Spectrosc Radiat Transf 10:909-928. doi:10.1016/0022-4073(70)90033-6

House LL (1970b) The resonance fluorescence of polarized radiation. II. Scattering in the normal Zeeman triplet $\left(J=0-J^{\prime \prime}=1\right)$. J Quant Spectrosc Radiat Transf 10:1171-1189. doi:10.1016/00224073(70)90002-6

House LL (1971) The resonance fluorescence of polarized radiation. III. The Stokes parameter and circular polarization formulation of the scattering redistribution function. J Quant Spectrosc Radiat Transf 11:367-383. doi:10.1016/0022-4073(71)90121-X

House LL, Baur TG, Hull HK (1975) Initial operation of a scanning Stokes polarimeter. Sol Phys 45:495500. doi:10.1007/BF00158465

Howard R, Stenflo JO (1972) On the filamentary nature of solar magnetic fields. Sol Phys 22:402-417. doi:10.1007/BF00148705

Illing RME, Landman DA, Mickey DL (1975) Broad-band circular polarization of sunspots-Spectral dependence and theory. Astron Astrophys 41:183-185

Ivanov VV (1991) Analytical methods of line formation theory-are they still alive. In: NATO ASIC proc 341: Stellar atmospheres-beyond classical models, p 81

Jiang J, Cameron RH, Schmitt D, Işık E (2013) Modeling solar cycles 15 to 21 using a flux transport dynamo. Astron Astrophys 553:A128. doi:10.1051/0004-6361/201321145

Jones HP, Giovanelli RG (1983) Magnetic canopies in unipolar regions. Sol Phys 87:37-42. doi:10.1007/BF00151157

Keller CU (1992) Resolution of magnetic flux tubes on the Sun. Nature 359:307. doi:10.1038/359307a0

Khlystova AI, Sokoloff DD (2009) Toroidal magnetic field of the Sun from data on Hale-rule-violating sunspot groups. Astron Rep 53:281-285. doi:10.1134/S106377290903010X

Khomenko E, Collados M (2007) On the Stokes V amplitude ratio as an indicator of the field strength in the solar internetwork. Astrophys J 659:1726-1735. doi:10.1086/512098

Kleint L, Berdyugina SV, Shapiro AI, Bianda M (2010) Solar turbulent magnetic fields: surprisingly homogeneous distribution during the solar minimum. Astron Astrophys 524:A37. doi:10.1051/0004$6361 / 201015285$

Knaack R, Stenflo JO (2005) Spherical harmonic decomposition of solar magnetic fields. Astron Astrophys 438:349-363. doi:10.1051/0004-6361:20052765

Kolmogorov A (1941) The local structure of turbulence in incompressible viscous fluid for very large Reynolds' numbers. Dokl Akad Nauk SSSR 30:301-305

Kosugi T, Matsuzaki K, Sakao T, Shimizu T, Sone Y, Tachikawa S, Hashimoto T, Minesugi K, Ohnishi A, Yamada T, Tsuneta S, Hara H, Ichimoto K, Suematsu Y, Shimojo M, Watanabe T, Shimada S, Davis JM, Hill LD, Owens JK, Title AM, Culhane JL, Harra LK, Doschek GA, Golub L (2007) The Hinode (Solar-B) mission: an overview. Sol Phys 243:3-17. doi:10.1007/s11207-007-9014-6

Kuhn JR, Harrington DM, Lin H, Berdyugina SV, Trujillo-Bueno J, Keil SL, Rimmele T (eds) (2011) In: Solar polarization 6. Astronomical society of the pacific conference series, vol 437 
Lagg A, Solanki SK, Riethmüller TL, Martínez Pillet V, Schüssler M, Hirzberger J, Feller A, Borrero JM, Schmidt W, del Toro Iniesta JC, Bonet JA, Barthol P, Berkefeld T, Domingo V, Gandorfer A, Knölker M, Title AM (2010) Fully resolved quiet-sun magnetic flux tube observed with the SUNRISE/IMAX instrument. Astrophys J Lett 723:L164-L168. doi:10.1088/2041-8205/723/2/L164

Landi Degl'Innocenti E, Landolfi M (2004) Polarization in spectral lines. In: Astrophysics and space science library, vol 307. Kluwer Academic, Norwell

Larmor J (1919) How could a rotating body such as the Sun become a magnet? Rep - Br Assoc Adv Sci $87: 159-160$

Leighton RB (1969) A magneto-kinematic model of the solar cycle. Astrophys J 156:1-26. doi:10.1086/ 149943

Leroy JL, Ratier G, Bommier V (1977) The polarization of the D3 emission line in prominences. Astron Astrophys 54:811-816

Li J, Ulrich RK (2012) Long-term measurements of sunspot magnetic tilt angles. Astrophys J 758:115. doi:10.1088/0004-637X/758/2/115

Lites BW, Kubo M, Socas-Navarro H, Berger T, Frank Z, Shine R, Tarbell T, Title A, Ichimoto K, Katsukawa Y, Tsuneta S, Suematsu Y, Shimizu T, Nagata S (2008) The horizontal magnetic flux of the quiet-Sun internetwork as observed with the Hinode spectro-polarimeter. Astrophys J 672:12371253. doi: $10.1086 / 522922$

Maltby P, Avrett EH, Carlsson M, Kjeldseth-Moe O, Kurucz RL, Loeser R (1986) A new sunspot umbral model and its variation with the solar cycle. Astrophys J 306:284. doi:10.1086/164342

Martin SF, Harvey KL (1979) Ephemeral active regions during solar minimum. Sol Phys 64:93-108. doi:10.1007/BF00151118

Moruzzi G, Strumia F (eds) (1991) The Hanle effect and level-crossing spectroscopy. Plenum, New York

Nagata S, Tsuneta S, Suematsu Y, Ichimoto K, Shimizu T, Yokoyama T, Tarbell TD, Lites BW, Shine RA, Berger TE, Title AM, Bellot Rubio LR, Orozco Suárez D (2008) Formation of solar magnetic flux tubes with kilogauss field strength induced by convective instability. Astrophys J Lett 677:L145L147. doi:10.1086/588026

Nagendra KN, Stenflo JO (eds) (1999) In: Solar polarization 2. Astrophysics and space science library, vol 243

Öhman Y (1929) Astronomical consequences of the polarization of fluorescence. Mon Not R Astron Soc 89:479-482

Omont A, Smith EW, Cooper J (1973) Redistribution of resonance radiation. 11. The effect of magnetic fields. Astrophys J 182:283-300. doi:10.1086/152136

Orozco Suárez D, Bellot Rubio LR, del Toro Iniesta JC, Tsuneta S, Lites BW, Ichimoto K, Katsukawa Y, Nagata S, Shimizu T, Shine RA, Suematsu Y, Tarbell TD, Title AM (2007) Quiet-Sun internetwork magnetic fields from the inversion of Hinode measurements. Astrophys J Lett 670:L61-L64. doi:10.1086/524139

Parker EN (1955) Hydromagnetic dynamo models. Astrophys J 122:293. doi:10.1086/146087

Parker EN (1978) Hydraulic concentration of magnetic fields in the solar photosphere. VI. Adiabatic cooling and concentration in downdrafts. Astrophys J 221:368-377. doi:10.1086/156035

Parnell CE, DeForest CE, Hagenaar HJ, Johnston BA, Lamb DA, Welsch BT (2009) A power-law distribution of solar magnetic fields over more than five decades in flux. Astrophys J 698:75-82. doi:10.1088/0004-637X/698/1/75

Petrovay K, Szakaly G (1993) The origin of intranetwork fields: a small-scale solar dynamo. Astron Astrophys 274:543

Pietarila Graham J, Danilovic S, Schüssler M (2009) Turbulent magnetic fields in the quiet Sun: implications of Hinode observations and small-scale dynamo simulations. Astrophys J 693:1728. doi:10.1088/0004-637X/693/2/1728

Povel H (1995) Imaging Stokes polarimetry with piezoelastic modulators and charge-coupled-device image sensors. Opt Eng 34:1870-1878

Povel HP (2001) Ground-based instrumentation for solar magnetic field studies, with special emphasis on the zurich imaging polarimeters ZIMPOL-I and II. In: Mathys G, Solanki SK, Wickramasinghe DT (eds) Magnetic fields across the Hertzsprung-Russell diagram. Astronomical society of the pacific conference series, vol 248, pp 543-552

Richardson RS (1948) Sunspot groups of irregular magnetic polarity. Astrophys J 107:78-93

Scherrer PH, Bogart RS, Bush RI, Hoeksema JT, Kosovichev AG, Schou J, Rosenberg W, Springer L, Tarbell TD, Title A, Wolfson CJ, Zayer I (MDI Engineering Team) (1995) The solar oscillations investigation-Michelson doppler imager. Sol Phys 162:129-188. doi:10.1007/BF00733429 
Scherrer PH, Schou J, Bush RI, Kosovichev AG, Bogart RS, Hoeksema JT, Liu Y, Duvall TL, Zhao J, Title AM, Schrijver CJ, Tarbell TD, Tomczyk S (2012) The Helioseismic and Magnetic Imager (HMI) investigation for the Solar Dynamics Observatory (SDO). Sol Phys 275:207-227. doi: $10.1007 / \mathrm{s} 11207-011-9834-2$

Schou J, Scherrer PH, Bush RI, Wachter R, Couvidat S, Rabello-Soares MC, Bogart RS, Hoeksema JT, Liu Y, Duvall TL, Akin DJ, Allard BA, Miles JW, Rairden R, Shine RA, Tarbell TD, Title AM, Wolfson CJ, Elmore DF, Norton AA, Tomczyk S (2012) Design and ground calibration of the Helioseismic and Magnetic Imager (HMI) instrument on the Solar Dynamics Observatory (SDO). Sol Phys 275:229259. doi:10.1007/s11207-011-9842-2

Schrijver CJ, Harvey KL (1994) The photospheric magnetic flux budget. Sol Phys 150:1-18. doi:10.1007/ BF00712873

Schüssler M, Baumann I (2006) Modeling the Sun's open magnetic flux. Astron Astrophys 459:945-953. doi:10.1051/0004-6361:20065871

Shapiro AI, Fluri DM, Berdyugina SV, Bianda M, Ramelli R (2011) NLTE modeling of Stokes vector center-to-limb variations in the CN violet system. Astron Astrophys 529:A139. doi:10.1051/ 0004-6361/200811299

Sheeley NR Jr, DeVore CR, Boris JP (1985) Simulations of the mean solar magnetic field during sunspot cycle 21. Sol Phys 98:219-239. doi:10.1007/BF00152457

Snik F, de Wijn AG, Ichimoto K, Fischer CE, Keller CU, Lites BW (2010) Observations of solar scattering polarization at high spatial resolution. Astron Astrophys 519:A18. doi:10.1051/0004-6361/ 201014500

Sokoloff D, Khlystova AI (2009) The solar dynamo in the light of the distribution of various sunspot magnetic classes over butterfly diagram. Astron Nachr 331:82-87. doi:10.1002/asna.200911300

Solanki SK (1993) Small-scale solar magnetic fields—an overview. Space Sci Rev 63:1-188. doi:10.1007/ BF00749277

Spruit HC (1977) Magnetic flux tubes and transport of heat in the convection zone of the Sun. PhD thesis, Univ Utrecht

Spruit HC (1979) Convective collapse of flux tubes. Sol Phys 61:363-378

Spruit HC, Zweibel EG (1979) Convective instability of thin flux tubes. Sol Phys 62:15-22

Steenbeck M, Krause F (1969) Zur Dynamotheorie stellarer und planetarer Magnetfelder I. Berechnung sonnenähnlicher Wechselfeldgeneratoren. Astron Nachr 291:49-84

Steiner O (2000) The formation of asymmetric Stokes V profiles in the presence of a magnetopause. Sol Phys 196:245-268

Steiner O (2010) Magnetic coupling in the quiet solar atmosphere. In: Hasan SS, Rutten RJ (eds) Magnetic coupling between the interior and atmosphere of the Sun, pp 166-185. doi:10.1007/978-3-64202859-5-13

Stenflo JO (1970) Hale's attempts to determine the Sun's general magnetic field. Sol Phys 14:263-273. doi: $10.1007 / \mathrm{BF} 00221312$

Stenflo JO (1971) The interpretation of magnetograph results: the formation of absorption lines in a magnetic field. In: Howard R (ed) Solar magnetic fields. IAU symposium, vol 43, pp 101-129

Stenflo JO (1973) Magnetic-field structure of the photospheric network. Sol Phys 32:41-63. doi:10.1007/BF00152728

Stenflo JO (1976) Small-scale solar magnetic fields. In: Bumba V, Kleczek J (eds) Basic mechanisms of solar activity. IAU symposium, vol 71, pp 69-99

Stenflo JO (1978) Resonance-line polarization. III. The Hanle effect in a compact non-LTE radiative transfer formulation. Astron Astrophys 66:241-248

Stenflo JO (1980) Resonance-line polarization. V. Quantum-mechanical interference between states of different total angular momentum. Astron Astrophys 84:68-74

Stenflo JO (1982) The Hanle effect and the diagnostics of turbulent magnetic fields in the solar atmosphere. Sol Phys 80:209-226

Stenflo JO (1987) Observational constraints on a 'hidden', turbulent magnetic field of the Sun. Sol Phys 114:1-19

Stenflo JO (1989) Small-scale magnetic structures on the Sun. Astron Astrophys Rev 1:3-48

Stenflo JO (1994a) Cycle patterns of the axisymmetric magnetic field. In: Rutten RJ, Schrijver CJ (eds) Solar surface magnetism, pp 365-377

Stenflo JO (1994b) Solar magnetic fields-polarized radiation diagnostics. Kluwer Academic, Dordrecht

Stenflo JO (1999) Solar magnetism and the second solar spectrum: future directions. In: Nagendra KN, Stenflo JO (eds) Polarization, astrophysics and space science library, vol 243, pp 1-16 
Stenflo JO (2003) Scattering polarization in magnetic fields: anomalies, surprises and enigmas. In: Trujillo Bueno J, Sánchez Almeida J (eds) Astronomical society of the pacific conference series, vol 307, pp 385-398

Stenflo JO (2004) The new world of scattering physics seen by high-precision imaging polarimetry (with 14 figures). In: Schielicke RE (ed) Reviews in modern astronomy, vol 17, pp 269-296

Stenflo JO (2010a) Distribution functions for magnetic fields on the quiet Sun. Astron Astrophys 517:A37. doi:10.1051/0004-6361/200913972

Stenflo JO (2010b) Measuring the hidden aspects of solar magnetism. In: Hasan SS, Rutten RJ (eds) Magnetic coupling between the interior and atmosphere of the Sun, pp 101-117. doi:10.1007/978-3642-02859-5-8

Stenflo JO (2011) Collapsed, uncollapsed, and hidden magnetic flux on the quiet Sun. Astron Astrophys 529:A42. doi:10.1051/0004-6361/201016275

Stenflo JO (2012a) Basal magnetic flux and the local solar dynamo. Astron Astrophys 547:A93. doi:10. $1051 / 0004-6361 / 201219833$

Stenflo JO (2012b) Scaling laws for magnetic fields on the quiet Sun. Astron Astrophys 541:A17. doi:10. $1051 / 0004-6361 / 201218939$

Stenflo JO (2013) Horizontal or vertical magnetic fields on the quiet Sun. Angular distributions and their height variations. Astron Astrophys 555:A132. doi:10.1051/0004-6361/201321608

Stenflo JO, Guedel M (1988) Evolution of solar magnetic fields-modal structure. Astron Astrophys 191:137-148

Stenflo JO, Keller CU (1996) New window for spectroscopy. Nature 382:588. doi:10.1038/382588a0

Stenflo JO, Keller CU (1997) The second solar spectrum. A new window for diagnostics of the Sun. Astron Astrophys 321:927-934

Stenflo JO, Kosovichev AG (2012) Bipolar magnetic regions on the Sun: global analysis of the SOHO/MDI data set. Astrophys J 745:129. doi:10.1088/0004-637X/745/2/129

Stenflo JO, Nagendra KN (eds) (1996) In: Solar polarization 1. Solar phys, vol 164

Stenflo JO, Vogel M (1986) Global resonances in the evolution of solar magnetic fields. Nature 319:285290

Stenflo JO, Twerenbold D, Harvey JW (1983a) Coherent scattering in the solar spectrum-Survey of linear polarization in the range 3165-4230 А. Astron Astrophys Suppl Ser 52:161-180

Stenflo JO, Twerenbold D, Harvey JW, Brault JW (1983b) Coherent scattering in the solar spectrumSurvey of linear polarization in the range 4200-9950 ̊. Astron Astrophys Suppl Ser 54:505-514

Stenflo JO, Solanki S, Harvey JW, Brault JW (1984) Diagnostics of solar magnetic fluxtubes using a Fourier transform spectrometer. Astron Astrophys 131:333-346

Stenflo JO, Keller CU, Gandorfer A (1998) Differential Hanle effect and the spatial variation of turbulent magnetic fields on the Sun. Astron Astrophys 329:319-328

Stenflo JO, Demidov ML, Bianda M, Ramelli R (2013) Calibration of the 6302/6301 Stokes V line ratio in terms of the 5250/5247 ratio. Astron Astrophys 556:A113. doi:10.1051/0004-6361/201321749

Stepanov VE, Severny AB (1962) A photoelectric method for measurements of the magnitude and direction of the solar magnetic field. Izv Krym Astrofiz Obs 28:166-193

Suematsu Y, Tsuneta S, Ichimoto K, Shimizu T, Otsubo M, Katsukawa Y, Nakagiri M, Noguchi M, Tamura T, Kato Y, Hara H, Kubo M, Mikami I, Saito H, Matsushita T, Kawaguchi N, Nakaoji T, Nagae K, Shimada S, Takeyama N, Yamamuro T (2008) The solar optical telescope of Solar-B (Hinode): the optical telescope assembly. Sol Phys 249:197-220. doi:10.1007/s11207-008-9129-4

Thompson MJ, Balogh A, Culhane JL, Nordlund Å, Solanki SK, Zahn JP (eds) (2009) The origin and dynamics of solar magnetism. Springer, Berlin. doi:10.1007/978-1-4419-0239-9

Tlatov A, Illarionov E, Sokoloff D, Pipin V (2013) A new dynamo pattern revealed by the tilt angle of bipolar sunspot groups. Mon Not R Astron Soc 432:2975-2984. doi:10.1093/mnras/stt659

Trujillo Bueno J (2001) Atomic polarization and the hanle effect. In: Sigwarth M (ed) Advanced solar polarimetry-theory, observation, and instrumentation. Astronomical society of the pacific conference series, vol 236, pp 161-195. arXiv:astro-ph/0202328

Trujillo Bueno J, Sanchez Almeida J (eds) (2003) In: Solar polarization 3. Astronomical society of the pacific conference series, vol 307

Trujillo Bueno J, Landi Degl'Innocenti E, Collados M, Merenda L, Manso Sainz R (2002) Selective absorption processes as the origin of puzzling spectral line polarization from the Sun. Nature 415:403406. arXiv:astro-ph/0201409

Trujillo Bueno J, Shchukina N, Asensio Ramos A (2004) A substantial amount of hidden magnetic energy in the quiet Sun. Nature 430:326-329. doi:10.1038/nature02669. arXiv:astro-ph/0409004 
Tsuneta S, Ichimoto K, Katsukawa Y, Nagata S, Otsubo M, Shimizu T, Suematsu Y, Nakagiri M, Noguchi M, Tarbell T, Title A, Shine R, Rosenberg W, Hoffmann C, Jurcevich B, Kushner G, Levay M, Lites B, Elmore D, Matsushita T, Kawaguchi N, Saito H, Mikami I, Hill LD, Owens JK (2008) The solar optical telescope for the Hinode mission: an overview. Sol Phys 249:167-196. doi:10.1007/s11207008-9174-Z

Unno W, Ando H (1979) Instability of a thin magnetic tube in the solar atmosphere. Geophys Astrophys Fluid Dyn 12:107-115. doi:10.1080/03091927908242679

Vögler A, Schüssler M (2007) A solar surface dynamo. Astron Astrophys 465:L43-L46. doi:10.1051/ 0004-6361:20077253. arXiv:astro-ph/0702681

Wang YM, Sheeley NR Jr (1989) Average properties of bipolar magnetic regions during sunspot cycle 21. Sol Phys 124:81-100. doi:10.1007/BF00146521

Wittmann A (1973) Computation and observation of Zeeman multiplet polarization in Fraunhofer lines. I: Photographic measurement of Stokes parameters. Sol Phys 33:107-118. doi:10.1007/BF00152383

Zirin H (1987) Weak solar fields and their connection to the solar cycle. Sol Phys 110:101-107. doi:10.1007/BF00148205 